\title{
Cortical Point-Spread Function and Long-Range Lateral Interactions Revealed by Real-Time Optical Imaging of Macaque Monkey Primary Visual Cortex
}

\author{
Amiram Grinvald, ${ }^{1,2,3}$ Edmund E. Lieke, ${ }^{2, a}$ Ron D. Frostig, ${ }^{2, b}$ and Rina Hildesheim ${ }^{2}$ \\ ${ }^{1}$ The Weizmann Institute of Science, Rehovot, 76100 Israel, Laboratory of Neurobiology, ${ }^{2}$ The Rockefeller University, \\ New York, New York 10021, and ${ }^{3}$ IBM Research Division, Yorktown Heights, New York 10598
}

Processing of retinal images is carried out in the myriad dendritic arborizations of cortical neurons. Such processing involves complex dendritic integration of numerous inputs, and the subsequent output is transmitted to multiple targets by extensive axonal arbors. Thus far, details of this intricate processing remained unexaminable. This report describes the usefulness of real-time optical imaging in the study of population activity and the exploration of cortical dendritic processing. In contrast to single-unit recordings, optical signals primarily measure the changes in transmembrane potential of a population of neuronal elements, including the often elusive subthreshold synaptic potentials that impinge on the extensive arborization of cortical cells.

By using small visual stimuli with sharp borders and realtime imaging of cortical responses, we found that shortly after its onset, cortical activity spreads from its retinotopic site of initiation, covering an area at least 10 times larger, in upper cortical layers. The activity spreads at velocities from 100 to $250 \mu \mathrm{m} / \mathrm{msec}$. Near the $V_{1} / V_{2}$ border the direct activation is anisotropic and we detected also anisotropic spread; the "space constant" for the spread was $\sim 2.7 \mathrm{~mm}$ parallel to the border and $\sim 1.5 \mathrm{~mm}$ along the perpendicular axis. In addition, we found cortical interactions between cortical activities evoked by a small "center stimulus" and by large "surround stimuli" positioned outside the classical receptive field. All of the surround stimuli used suppressed the cortical response to the center stimulus. Under some stimulus conditions iso-orientation suppression was more pronounced than orthogonal-orientation suppression. The orientation dependence of the suppression and its dependency on the size of some specific stimuli indicate that at least part of the center surround inhibitory interaction was of cortical origin.

\footnotetext{
Received Dec. 9, 1992; revised Sept. 24, 1993; accepted Oct. 4, 1993.

We thank Drs. Torsten Wiesel, Charles Gilbert, and Dan Ts'o for their comments and encouragement throughout the work; Lawrence Katz for participation in the slice experiments; Moshe Abeles, Ehud Kaplan, Rafi Malach, Amos Arieli, and one anonymous referee for their constructive comments on the manuscript; and Gene Ratzlaff, Kaare Christian, Doron Shoham, and Steve Leytus for excellent technical assistance. This work was supported by IBM, NIH Grant NS 14716 and the Schilling Foundation.

Correspondence should be addressed to Amiram Grinvald, Department of Neurobiology, the Weizmann Institute of Science, Rehovot, 76100, Israel.

aPresent address: Freie Universität Berlin, Abteilung für Neurobiologie, Königin-Luise-Strasse 28/30, D-1000 Berlin 33, Germany.

'Present address: Department of Psychobiology, University of California, Irvine, CA 92717.
}

Copyright (c) 1994 Society for Neuroscience $0270-6474 / 94 / 142545-24 \$ 05.00 / 0$
The findings reported here raise the possibility that distributed processing over a very large cortical area plays a major role in the processing of visual information by the primary visual cortex of the primate.

[Key words: vision, lateral connections, point-spread function, functional architecture; voltage-sensitive dyes, imaging]

Individual cortical cells process information originating from thousands of other neurons that are often distributed over large distances. It is therefore not easy to explore mechanisms underlying cortical processing with traditional electrophysiological recording techniques. Furthermore, the inputs to a single cortical cell are initially processed, passively or actively, by the dendritic arborization. This dendritic processing usually remains elusive when using electrical recordings in vivo. Cortical processing also depends on the exact timing of the multiple inputs. Therefore, what appear to be needed are additional tools that can detect electrical activity of many neurons including their spectacular arborization, offering both high temporal and spatial resolution, simultaneously. This report focuses on the application of voltage-sensitive dyes to explore a few of the questions that can benefit from the direct imaging of activity of millions of cortical neurons. Two forthcoming articles will focus on the exploration of the spatiotemporal properties of neuronal populations in relation to those of selected single units (A. Arieli, D. Shoham and A. Grinvald, unpublished observations; D. Shoham, S. Ullman, A. Arieli, and A. Grinvald, unpublished observations) Below we discuss the scientific background related to the questions explored here.

The first question this manuscript addresses is whether retinotopic borders are also sharp in cortical layers other than the input layer. Electrophysiological mapping studies of the primary visual cortex in the macaque monkey have revealed its retinotopic organization (Talbot and Marshal, 1941; Daniel and Whitteridge, 1961; Hubel and Wiesel, 1974; Dow et al., 1981, 1985; Van Essen et al., 1984). A more direct approach in the study of the retinotopic organization of primary visual cortex has been introduced by Tootell and his colleagues, utilizing 2-deoxyglucose (2-DG) imaging experiments (Tootell et al., 1982, 1988). These 2-DG studies clearly showed that in layer IV, the input layer, remarkably sharp borders of cortical activity are produced by visual stimuli with well-defined borders. On the other hand, anatomical and physiological investigations in cat and monkey striate cortex have uncovered the existence of longrange horizontal connections, extending in some extreme cases up to $8 \mathrm{~mm}$ parallel to the cortical surface (Fisken et al., 1975; 
Creutzfeldt et al., 1977; Gilbert and Wiesel, 1979, 1983, 1989; Rockland and Lund, 1982; Livingstone and Hubel, 1984; Martin and Whitteridge, 1984; Blasdel et al., 1985; Fitzpatrick et al., 1985). If most intrinsic horizontal axons are indeed several millimeters long, one would expect that focal cortical activation by sensory input would spread over distances much larger than predicted from retinotopic data for the input layer. Since such a large lateral spread of activity in upper and lower cortical layers has not been reported in the 2-DG studies, there is a need to reconcile the apparent contradiction between $2-D G$ investigations on the one hand and anatomical studies of the axonal morphology of cortical neurons on the other. The data described in this report resolve this apparent contradiction.

Most cellular studies of intrinsic lateral connections have used traditional receptive field analysis to study the underlying mechanisms. In particular, they have addressed the question of which area(s) of the visual field affects the firing properties of individual cortical neurons. McIlwain (1986) has pointed out that only a few studies have investigated the inverse problem, that is, the mapping transformation of retinal activity into cortical activity, referred to as the cortical point-image. To determine the cortical point-image, instead of asking, "What are the points in the visual field that a single cell "sees"?" the question becomes "Where are the cortical cells that 'see' this retinal point?" (Fischer, 1973; Albus, 1975; McIlwain, 1975; Dow et al., 1981, 1985; Van Essen et al., 1984; Tootell et al., 1988). Since electrical recordings are only a one-dimensional mapping tool, they are not optimal for such mapping as functions of various stimulus attributes: time, state of the animal, and so forth. Furthermore, whenever the long-range lateral connections give rise mostly to subthreshold dendritic activation, such spreading may be missed by classical recording techniques. We therefore decided to reexamine the spread of cortical responses using real-time optical imaging.

Several psychophysical experiments have indeed suggested that such spread may exist by demonstrating the remarkable effects on visual perception of the texture of visual stimuli in the area surrounding, and extending far beyond, the classical receptive field of cortical neurons (Campbell and Kulikowski, 1966; Julesz et al., 1976; Sagi and Julesz, 1985; Cannon and Fullenkamp, 1991). Electrophysiological studies in cat primary visual cortex also revealed interactions between responses to two adjacent visual stimuli beyond the classical receptive field, and these may be related to the psychophysical studies discussed above and to the anatomic findings of long-range intrinsic connections. In most cases these interactions were inhibitory; however, disinhibition and facilitatory effects have also been reported (Jones, 1970; Creutzfeldt et al., 1971; Blakemore and Tobin, 1972; Maffei and Fiorentini, 1976; Fries et al., 1977; Hammond and MacKay, 1977, 1981; Nelson and Frost, 1978, 1985; Orban et al., 1987). Some of these interactions may have originated in processing stations of the visual system prior to the cortcx, as previously reported for cat and monkey retina and LGN (Mcllwain, 1966; Kruger and Fischer, 1973; Kruger, 1977; Kaplan and Shapley, 1989). In the primate visual cortex, however, long-range interactions were initially observed only in higher visual areas (e.g., Zeki, 1983; Allman et al., 1985). Only recently were similar interactions also found in primary visual cortex of the primate (Van Essen et al., 1989; Born and Toolell, 1991; Knierim and Van Essen, 1992).

The functional significance of the long-range lateral connections has remained controversial. From extracellular single-unit recordings of spike activity and cross-correlation analysis, Ts'o et al. (1986) and Ts'o and Gilbert (1988) demonstrated longrange excitatory interactions between cortical cells with similar receptive field properties. However, Hata et al. (1988) demonstrated inhibitory horizontal connections in cat striate cortex. Therefore, the question of whether the primary effect of horizontal connections is excitatory or inhibitory has not yet been resolved. A serious difficulty with single-unit recording is its inability to detect cortical synaptic potentials; thus, critical features of cortical processing may go undetected (Brooks and Jung, 1973). It seemed, therefore, that a more complete understanding of synaptic action at the level of local cortical circuits may be achieved by intracellular studies. Li et al. (1960) first demonstrated postsynaptic potentials of both cxcitatory (EPSP) and inhibitory (IPSP) nature in striate cortex. More recent intracellular experiments in cat cortex have revealed both iso-orientation EPSPs and IPSPs related to orientation selectivity in the visual cortex (Ferster, 1986, 1988; Douglas et al., 1991; see Ferster and Koch, 1987, for review). Nevertheless, intracellular cortical recordings are difficult and the notorious sampling problems have complicated the interpretation of the data. Real-time optical imaging in vivo can help resolve some of these difficulties.

In vivo real-time optical imaging of sensory-evoked activity (Grinvald et al., 1984; Orbach et al., 1985) is based on the use of voltage-sensitive dyes (Tasaki et al., 1968; Salzberg et al., 1973; Cohen et al., 1974, Grinvald et al., 1977; Waggoner and Grinvald, 1977; Loew et al., 1985). These dyes act as molecular transducers, transforming changes in membrane potential into optical signals. Real-time optical imaging thus offers (1) simultaneous recording of changes in transmembrane potentials from many sites with millisecond time resolution, (2) the detection of action potentials in very small nonmyelinated axons, and (3) the detection of subthreshold potentials in the extensive dendritic arbor. These features should allow the direct imaging of the cortical point-spread function, emphasizing the often elusive electrical activity in the cortical neuropil. [For technical reviews, sec Cohen and Lesher (1986), DeWeer and Salzberg (1986), Loew (1987), Orbach (1987), Grinvald et al. (1988), and Lieke et al. (1989).]

Preliminary results have previously been presented in abstract form (Lieke et al., 1988) and in chapters of "proceedings" (Grinvald et al., 1989, 1991b); portions of Figures 8, 9, 11, and 1315 were published in modified form in these chapters.

\section{Materials and Methods}

Several technical problems were not solved during the first optical imaging experiments performed on cat visual cortex (Orbach et al., 1985), and substantial improvements were therefore essential. Furthermore, currently there may be a confusion between various types of optical signals. Since the proper interpretation of the optical signals has required a multidisciplinary approach, this section attcmpts to describe the improvements offered by the technique and to discuss the interpretation of the voltage-sensitive dye signals in considerable detail. In addition, this section includes some experimental results whenever the results are related to the methodology rather than to the scientific questions addressed in this article.

Animals. Twenty-three adult monkeys, Macaca fascicularis, were used for the development of the technique including preliminary experiments and the testing of voltage-sensitive dyes that may be species specific. The experiments described in detail here used four additional moneys and employed the new macroscope described below. Monkeys were initially anesthetized with ketamine $\mathrm{HCl}$ in their cages $(10-20 \mathrm{mg} / \mathrm{kg}$, i.m.). They were then transferred to an operating table, anesthetized with sodium pentothal (initial dose of $10-20 \mathrm{mg} / \mathrm{kg}$ followed by continuous infusion of $1-3 \mathrm{mg} / \mathrm{kg} / \mathrm{hr}$, i.p.), paralyzed with vercuronium bromide (Norcuron, $0.1-0.2 \mathrm{mg} / \mathrm{kg} / \mathrm{hr}$, i.v.), and artificially respirated. 
Fifty-four cats were used to develop the methodology and test voltagesensitive dyes. Cats were anesthetized in an identical way as described above but they were paralyzed with succinylcholine $(10-30 \mathrm{mg} / \mathrm{kg} / \mathrm{hr})$. In addition, we used brain slices to test voltage-sensitive dyes. Slices were prepared as described elsewhere for rats (Grinvald et al., 1982b), cats (Katz, 1987), and monkeys (Katz et al., 1989).

To monitor the state of the animal and the level of anesthesia, the EEG, ECG, end-tidal $\mathrm{CO}_{2}$, and rectal temperature were continuously monitored throughout the experiment. Arterial blood pressure was also measured with standard blood pressure transducers connected to a patient monitor (HP model 78354A). The pupils of the eyes were dilated with $1 \%$ atropine. A retinoscope was used to determine the appropriate contact lens that would focus the eyes on a tangent screen $1.8 \mathrm{~m}$ away. The contact lenses used had a $3 \mathrm{~mm}$ artificial pupil. The foveal position of each eye was determined with a fundus camera. To monitor eye movements and to position the visual stimulus to stimulate the center of the optically imaged area, a tungsten electrode was placed at the center of the imaged cortical region. Stimuli were presented binocularly and a Risley prism in front of one eye was used to fuse the gaze of the two eyes. The eccentricity of the recording site was determined by measuring the distance between the electrically recorded receptive field and the position of the fovea projected on the screen by the fundus camera.

Craniotomy. An 18-mm-diameter craniotomy was performed with a trephine and the dura resected. The anterior border of the hole was at least $1 \mathrm{~mm}$ posterior to the lunate sulcus. This ensured that the entire area exposed was included within area V1, close to the V1/V2 border. Typically, the center of the hole was $4-9^{\circ}$ below the horizontal meridian and $1-3^{\circ}$ lateral to the vertical meridian.

Optical chamber. To minimize movements of the cortical surface due to heart pulsation and respiration, a stainless steel chamber, $20 \mathrm{~mm}$ in diameter, was attached to the skull with the aid of dental cement. A leak in the chamber would give rise to cortical edema. To prevent leaks from the chamber, the space between the skull and the stainless steel ring was sealed with dental wax, melted by a cauterizer. The chamber was then covered with a 1-mm-thick round cover glass, supported by a silicone O-ring. A stainless steel ring then pressed the cover glass against the O-ring and sealed the chamber. Two stainless steel tubings were attached to the chamber to provide an inlet and an outlet. They facilitated the perfusion of dye staining solution, artificial cerebrospinal fluid (CSF), or silicone oil (Dow Corning $20050 \mathrm{cSt}$ ). To prevent cortical edema, solutions were exchanged through the closed chamber only by gravity (pressure from $5-60 \mathrm{~cm}$ water). Without the sealed chamber, cortical movement severely compromised satisfactory optical recording.

Electrical recordings through the sealed optical chamber. In these experiments we combined single-unit recordings with optical recordings, through the transparent cover glass of the sealed chamber. To achieve this goal, a 3-mm-diameter hole was drilled in the cover glass, and a rubber gasket, tightly fitting the hole, was glued in place with acrylic and epoxy glues. To protect the tungsten electrode ( $1 \mathrm{M} \Omega$ resistance), it was inserted into a thin Teflon tubing (back side first) and then into a 26 gauge, $6-\mathrm{cm}$-long, sharpened syringe needle. The syringe needle was attached to a hydraulic manipulator and could easily penetrate the rubber gasket, while the electrode itself was protected inside the needle. After the needle penetrated the rubber gasket, the electrode was advanced manually until the recording tip extended 3-6 $\mathrm{mm}$ beyond the tip of the needle. The electrode was then locked to the needle and advanced under visual control, using the hydraulic manipulator. The tungsten electrode was connected to a differential amplifier. To record single- or multiple-unit activity, the output was bandpass filtered at $0.3-3 \mathrm{kHz}$. The local field potential (LFP) from the same electrode was filtered at $1-100 \mathrm{~Hz}$. Electrical recordings through the sealed chamber were very stable over several hours.

Lack of eve movements. Residual eye movements can pose a serious problem for the interpretation of the present experiments. In two experiments, eye position was monitored by single-unit recording before and after the experiments and was found stable. Furthermore, whenever eye movement occurred it was manifested in the data itself; in experiments with monkey M24, we noticed that a small residual drift in eye position could be readily detected as shifted activation patterns. A comparison of the activation patterns for interlaced experiments, including center and surround stimuli, proved to be a sensitive tool for the detection of eye movements in M24. Such shifts were not detected in data from the other monkeys. Thus, we conclude that residual eye movements were not a problem in the other short imaging $(<6 \mathrm{~min})$ sessions reported here.
Staining with voltage-sensitive dyes. In the present experiments we have used the new styryl dye designated RH-795. The dye structure and its synthesis is described in the Appendix. The dye solution was applied topically for $1-2 \mathrm{hr}$ at a concentration of $0.1 \mathrm{mg} / \mathrm{ml}$ in artificial CSF. We found that with this staining procedure this hydrophilic dye diffused into deep cortical layers and often stained the cortex all the way to the white matter. The staining of the upper cortical layers up to a depth of $1 \mathrm{~mm}$ was always more pronounced.

The macroscope. Conventional microscope objectives were not suitable for optically imaging the activity from the large cortical area of 6 $\times 6 \mathrm{~mm}$. Their small numerical aperture is not optimal for fluorescence measurements. For example, a $2 \times$ Zeiss objective has a numerical aperture of 0.08 . To minimize photodynamic damage (to be discussed below), the collection of emitted fluorescence should be as efficient as possible. Since we wanted to image large cortical areas, we constructed a custom macroscope made from commercially available $35 \mathrm{~mm}$ camera lenses (Ratzlaff and Grinvald, 1991). We used a back-to-back mounting of a $50 \mathrm{~mm}$ f: 1.2 lens and a $130 \mathrm{~mm}$ f: 1.8 lens. Since the macroscope had a numerical aperture of approximately 0.4 , the epi-illumination intensity via the macroscope was improved by fourfold. More significant is the fact that its efficiency of fluorescence collection was found to be 20 -fold better than that of the $2 \times$ Zeiss objective. Overall, this arrangement provided an 80-fold improvement in the detection of fluorescence emission, and allowed us to reduce the illumination intensity, either to enhance the signal-to-noise ratio by longer averaging sessions, or to perform many more experiments, or both. All of the experiments described in Results were obtained with the macroscope.

Visual stimulation. High-contrast black-and-white gratings were used as stimuli. The visual stimulator was developed by K. Christian and Dr. E. Ratzlaff at the Rockefeller University. It was based on an IBMAT computer equipped with an SGT Plus video graphics board (Number Nine Corporation, Cambridge, MA). The visual stimuli were displayed on a CRT screen in $60 \mathrm{~Hz}$, noninterlaced mode (Mitsubishi model C-6922-PK). The monitor screen was positioned at a distance of 160 $\mathrm{cm}$, and its brightness was set at medium range. Since the vertical syno of the monitor was not synchronized to the data acquisition, there was an approximately $17 \mathrm{msec}$ jitter between data acquisition and the onset of the drift of the visual stimulus. For stimulation of monkey striatc cortex we used a spatial frequency of 3-4 cycles/degree and a drifting velocity of $0.5-1$ degree/sec. which generated a temporal frequency modulation of approximately $3.5 \mathrm{~Hz}$ at each point on the retina. To stimulate a small restricted cortical patch we used small gratings that drifted within a restricted window. The smallest window chosen was $1^{\circ}$ $\times 0.5^{\circ}$. Smaller point stimuli were not used in this study because of signal-to-noise considerations.

Data acquisition. Figure 1 shows the setup for real-time optical imaging. A $6 \times 6 \mathrm{~mm}$ image of primary visual cortex was projected onto a 10 by 10 or a 12 by 12 photodiode array (Grinvald et al., 1981; Cohen and Lesher, 1986) with the aid of a macroscope having a magnification of $2.3 \times$. Each individual diode was connected to a three-stage amplifier The first stage was an $I-V$ converter with a feedback resistor of $10 \mathrm{M} \Omega$. The second stage included a sample and-hold amplifier followed by a differential amplifier. The sample-and-hold sampled the input just after the shutter was opened but before each trial. This DC level was then differentially subtracted from the incoming signal. This arrangement facilitated the subtraction of the large DC component of the detected fluorescence. The small remaining signal was then amplified by the third stage providing a selectable gain ranging from 1 to 4000 . The parallel output of the amplifiers was multiplexed by two 64 channel 12 bit A/D converters and was transferred via direct-memory-access interface (DMA) to the computer memory. A PDP $11 / 44$ computer, running the RT-11 version 5 operating system, controlled the visual stimulator and collected the optical and electrical data. This RT- 11 operating system could address much more memory than older versions and it facilitated the interlacing of four different stimulus conditions.

A video monitor screen showed an image of the exposed cortical area with a superimposed image of the diode array (not shown). The computerized stimulator described above was controlled in turn by the PDP. The time course of the evoked optical signal from the center of the array was displayed on line on a Tektronix 4012 display monitor. This online display was designed to facilitate manual (or automatic) rejection of noisy trials from the accumulated average. Noisy trials were those that included noise three times as large as the average noise. Such trials were rare $(0-5 \%)$ in the present experiments, however.

Data display. Data analysis was performed by the PDP 11/44 and an 


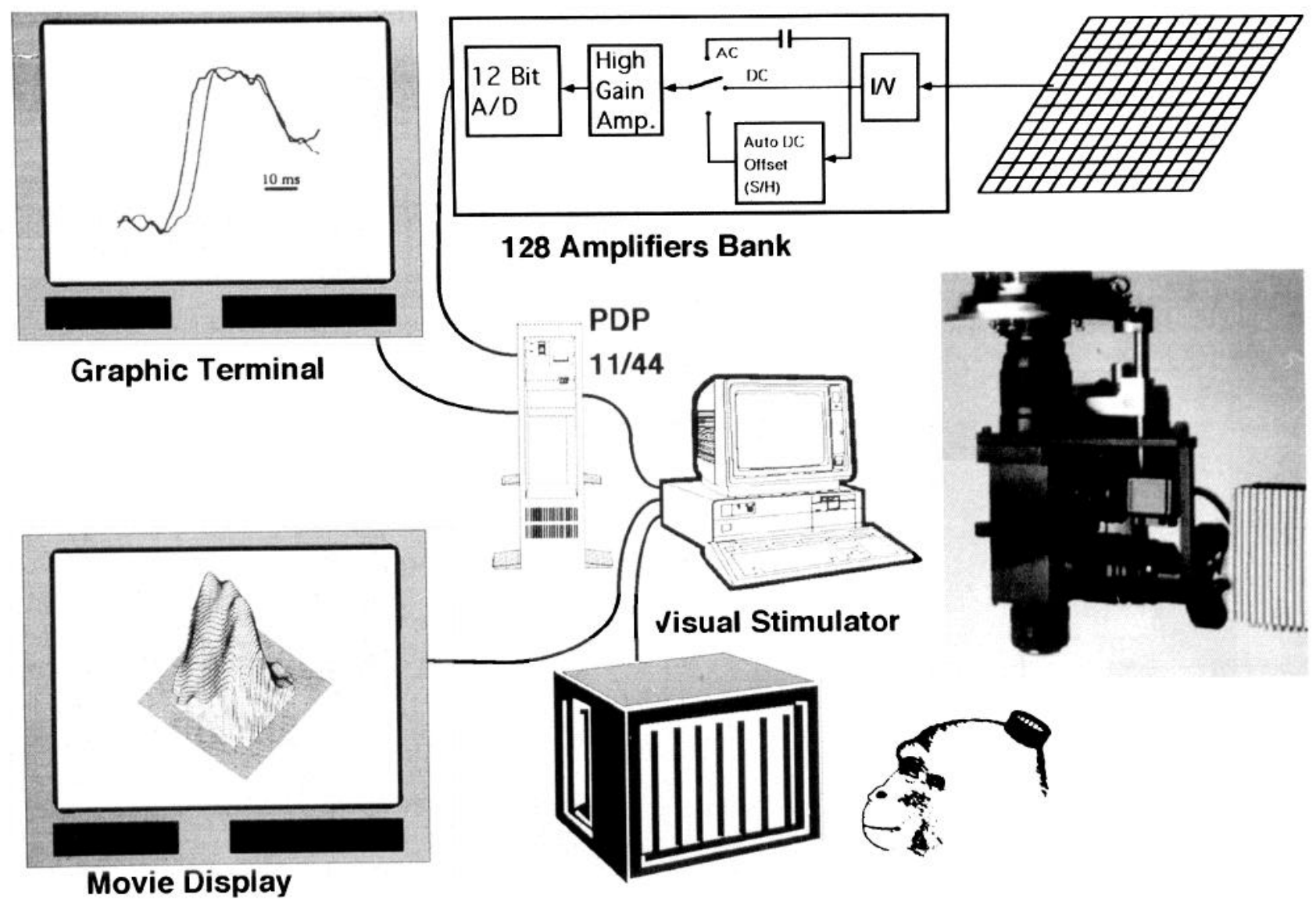

Figure 1. Optical apparatus for real-time imaging of electrical activity with a photodiode array. A $6 \times 6$ mm image of primary visual cortex was projected onto a 10 by 10 photodiode array with the aid of a macroscope (right side). Each individual diode was connected to a three-stage amplifier. The first stage was a $I-V$ converter. The second stage was connected to a sample-and-hold amplifier, thus facilitating the subtraction of the large DC component of the detected fluorescence. The small remaining DC component was then amplified by the third stage providing a gain ranging from 1 to 4000 . The parallel output of the amplifiers was transferred via DMA to the PDP 11/44 memory. Visual stimuli were programmed on a IBM-AT computer. The computerized stimulator was controlled by the PDP 11/44. The time course of the evoked optical signal from the center of the array was displayed on line on a Tektronix 4012 display monitor (top left). A surface plot program was used to convert the output of the diode array into a surface plot. Each plot was then used as a frame of a movie, which was displayed in slow motion on an RGB monitor (bottom left).

IBM PS2/80 or IBM-AT. A three-dimensional plot showing the amplitude of the activity as a function of position was plotted for each frame (sURFER package, Golden Software Inc.). These frames, referred to as surface plots, were combined into a movie that was displayed on an RGB monitor by an IBM-AT computer equipped with a video display board (Number Nine Computer Co., Cambridge, MA). To enhance the image, the surface plot used spliced interpolation (without smoothing). This means that if the original surface plot of the 10 by 10 image were superimposed on the surface plots shown here, all of the 100 points would lie precisely in the interpolated surface.

Imaging procedures. Cortical movements were not apparent in the sealed chamber, even when the cortex was viewed with a high-power microscope. However, systematic noise, time-locked to the heartbeat and the respiration, was at least as large as the visually evoked optical signal. To reduce the large noise from heart pulsation and respiration, data acquisition was triggered by the ECG (Grinvald et al., 1984; Orbach at al., 1985). Furthermore, the respirator was resynchronized to the ECG prior to every pair of trials. We essentially eliminated these very large biological contributions of noise, in two ways. First, we subtracted responses obtained with a visual stimulus from those without a stimulus. Second, to increase the reproducibility of the artifact created by the heartbeat, we stabilized the heart rate by an intramuscular injection of hexamethonium $(10 \mathrm{mg} / \mathrm{kg} / \mathrm{hr})$ and maintained the normal blood pressure, if needed, by injection of Vasoxyl (methoxamine hydrochloride; $0.1-0.2 \mathrm{mg} / \mathrm{kg} / \mathrm{hr}$ ). Hexamethonium acts as a sympathetic and parasympathetic blocker, while Vasoxyl is a vasoconstrictor with relatively minimal effect on the heart.

Figure $2 A$ illustrates the reproducibility of single sweep optical re- cordings obtained from monkey M16 by the procedure described above. Six superimposed trials are shown for each trace. Data acquisition was triggered by the ECG, and in every even-numbered trial a drifting grating was turned on and presented to the monkey. The surface recording of the field potential is shown above the trace of the stimulus. Two families of optical signals are shown above this trace, corresponding to trials with and without a stimulus. (Using this dye, depolarization gives rise to a fluorescence decrease. Therefore, the signal sign was inverted.) The top traces are the results of the subtraction of a trial where a visual stimulus was presented from a consecutive trial without a stimulus. The figure demonstrates that with the RH-795 dye, visually evoked cortical activity in monkey cortex could be detected without signal averaging.

Each set of experiments consisted of four interlaced stimulus conditions, and 20 trials were averaged for each condition. For different experiments different sets of four stimuli were chosen. The interval between stimuli was $2 \mathrm{sec}$. The optical imaging of the responses to four different stimuli took less than $6 \mathrm{~min}$. Figure $2 B$ shows the averaged optical signal and the averaged evoked potential corresponding to the single trials shown in Figure $2 A$. The onset of the optical response was delayed by $\sim 80 \mathrm{msec}$ relative to the onset of the visual stimulus, as expected from the retinal delay. The optical signal quickly returned to baseline after the visual stimulus disappeared. Although the optical recording is a DC recording and the electrical recording was bandpass filtered, the correlation between many peaks in the optical signals and peaks in the evoked potential (arrowheads) is evident.

Flashing of drifting gratings on the screen for a fraction of a second elicited the relatively large signals that could be detected without signal averaging as depicted by the example shown in Figure 2. However, to 
A

\section{Single trials}

\section{Optical \\ signal \\ (Corrected)}
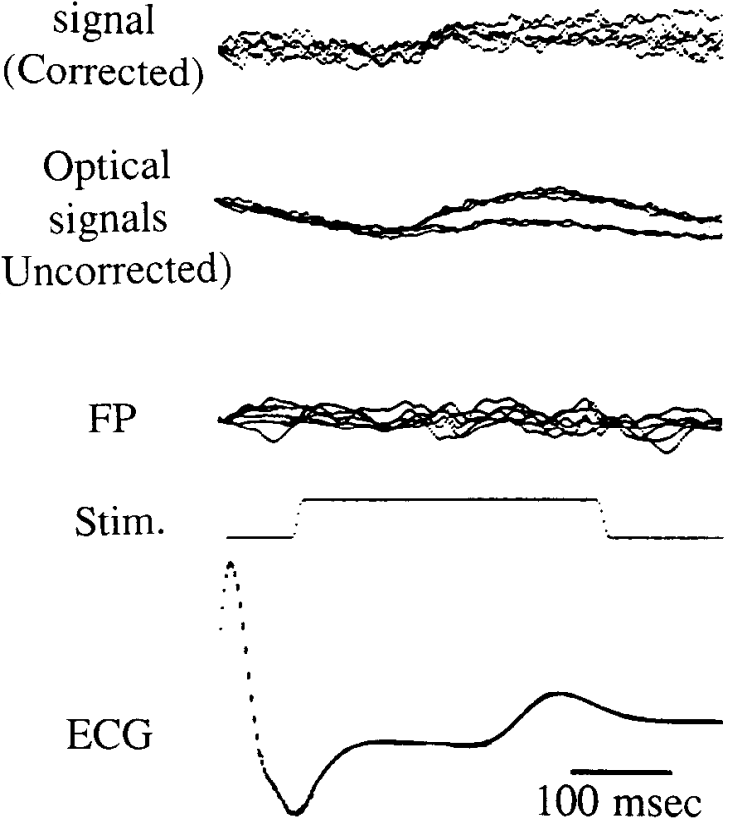

$\mathrm{B}$

\section{Averaged signals}

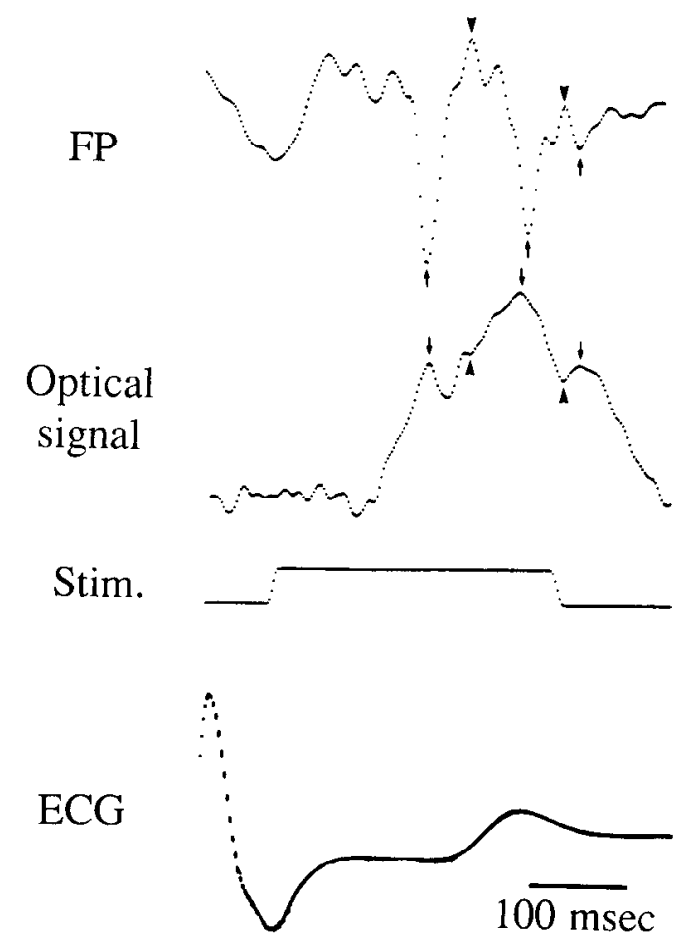

Figure 2. The noise reduction procedure for real-time optical imaging. $A$, Successive single trials are shown in each trace. The data acquisition was triggered by the electrocardiogram $(E C G)$. The bottom traces demonstrate the reproducibility of the ECG. The next set of traces above shows the time course of the visual stimulus. The traces above that show the electrical recordings of the visually evoked field potential $(F P)$. The next set of traces shows two families of optical signals: one obtained with and one without a visual stimulus, each recorded three times. The traces obtained without the stimulus (those with larger amplitude) show the time course of the heartbeat pulsation and were used to correct the stimulus-evoked optical signals (traces exhibit lower amplitude, since depolarization shows a decrease in fluorescence with this dye). The top trace shows six singletrial optical recordings after subtraction of the corresponding no-stimulus trials. These signals are inverted so that depolarization appears like a positive signal. The stimulus was a drifting grating, which was turned on at the times shown by the stimulus tracc. The cortex was stained with the dye RH-704 $(0.1 \mathrm{mg} / \mathrm{ml})$ for $1.5 \mathrm{hr}$ (structure shown in Fig. 18). $B$, The averaged signals of the results described in $A$. Twenty trials were averaged. The traces, starting from the bottom, are the ECG, stimulus, optical signal, and the field potential $(F P)$. Note that many peaks in the optical recording correspond to peaks in the electrically recorded evoked potential (arrows). See Materials and Methods for discussion. The fractional change in fluorescence signal was $5 \times 10^{-4}$. Here and in all other figures a decrease of fluorescence intensity is plotted in the upward direction, because styryl dye depolarization gives rise to a decrease in fluorescence. (At the beginning of the evoked potential trace there is a large amount of slow "noise" seen also in one of the single trials shown in $A$.)

minimize the contribution of on and off transients and to maximize the contributions of cortical responses selective to the orientation of the moving gratings, in all of the experiments reported in Results we used stationary gratings during the interstimulus interval instead of a blank screen. Thus, the evoked signal was the one obtained by subtracting the traces obtained during the presentation of a stationary grating from the corresponding signal evoked by drifting the same grating stimulus.

We were concerned whether the stationary stimuli used during the interstimulus interval would affect the results. Single-unit recordings could not detect a significant transient response to the stationary stimuli. Furthermore, since such stationary gratings also did not produce transient optical signals from a population of neurons monitored, the present stimulation/subtraction procedure seems adequate to optimize signals that were selective for orientation. An independent set of experiments, in which imaging based on intrinsic signals was used to map orientation domains in cat and monkey striate cortex, repeatedly confirmed that this procedure was better than the use of flashing gratings; the signalto-noise ratio in the resulting orientation map was better with standing/ drifting stimuli than with a blank screen replaced by a drifting grating.

What does the signal from the dye measure? Simultaneous intracellular and optical recordings from single cells have shown that styryl dyes measure the fast changes in membrane potential, identical to intracellular recordings (Grinvald et al., 1982a, 1983; Loew et al., 1985). The size of the optical signal is linearly related to the membrane area. Because the area of the membrane of both dendritic and axonal arbors of cortical cells is approximately 1000 -fold larger than that of the cell soma, optical recordings should give a large bias to dendritic and axonal activity. Therefore, optical recordings from neuronal populations in cortical tissue should be very different from both intracellular and field potential recordings and could show a picture of cortical activity not seen with these traditional electrical recording techniques.

Since many mixed neuronal elements are viewed by the same detector, the optically detected signal integrates the membrane potential changes from many different neuronal elements. These include both pre- and postsynaptic elements, cell somata, and even glial cell depolarization due to potassium accumulation (Konnerth and Orkand, 1986; Lev-Ram and Grinvald, 1986; Konnerth et al., 1987). Both field potential and optical recordings reflect population activity. However, in contrast with optical recordings, field potentials are affected by currents in the extracellular space. Thus, field potentials are not restricted to the site of changes in membrane potential, and may often spread in an unpredictable fashion, whereas optical signals are restricted to the site of membrane potential change. Therefore, real-time optical imaging provides a much better spatial resolution than electrical recording of field potentials.

To prove that the optical signals recorded from the visual cortex correspond to neuronal activity, we compared optical signals with electrical recordings, measured simultaneously. For example, Figure 3 shows 

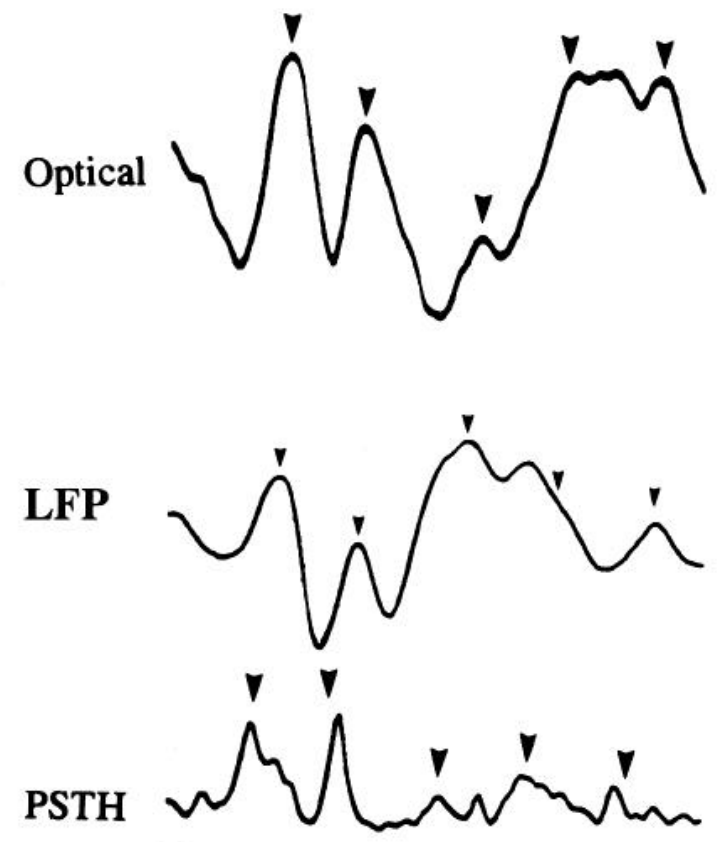

Stim.

\section{$100 \mathrm{msec}$}

Figure 3. Comparison between optical, LFP, and single-unit recordings. An averaged off response to a $500 \mathrm{msec}$ drifting grating is shown. The bottom trace shows the timing of the stimulus. The second trace is the smoothed PSTH of a single unit, recorded with a tungsten electrode (a total of 254 spikes were recorded). The next trace shows the local field potential $(L F P)$ recorded via the same electrode filtered at $1 \mathrm{~Hz}$ to $100 \mathrm{~Hz}$. The top trace shows the optical signal. The fractional change in fluorescence for the peaks of the optical signal was $3 \times 10^{-4}$ and 70 trials were averaged. The correspondence of various peaks is shown by the arrowheads. In this selected example there is a striking similarity between peaks of activity obtained by these very different recording techniques (see Materials and Methods).

the off response to a drifting grating, monitored by single-unit recording, a local field potential recording from the same electrode, and optical recordings by the diodes imaging the electrode recording site. To show the similarity between the timings of activity recorded by each of the different techniques, one can draw a grid of parallel lines or use timing markers producing a "timing ruler." We used the latter approach and labeled several large and a few small peaks on the optical signal (top trace) with arrowheads. Using small shifts of the entire timing ruler but without any shift of their relative latencies, the same timing marks (arrowheads) were positioned above the two electrical traces below, so that the first arrowhead was above the first peak of each recording. A correlation between the peaks of population activity, recorded by the two very different techniques, was observed. A similar relationship was also observed for the LFP and optical signals shown in Figure $2 B$. The similarity between the electrical recordings and the optical recordings clearly indicates that the signal from the dye is directly related to electrical activity. Additional evidence will be presented elsewhere (Arieli, Shoham, and Grinvald, unpublished observations). It is worth mentioning that both the local field potential and intracellular recordings emphasize activity of synaptic input to the cortex (Klee et al., 1965; Frost, 1968; Elul, 1972; Creutzfeldt and Houchin, 1974). Note, however, that the local field potential and the dye signal may have opposite polarities, depending on the depth of the sinks and sources of the field potential (for a thorough discussion, see Creutzfeldt and Houchin, 1974).

A nice correspondence was observed also for the activity of the unit illustrated here; that is, peaks seen in the PSTH (peristimulus time histogram) corresponded to peaks detected by the two recordings of

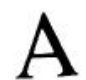

RH-773

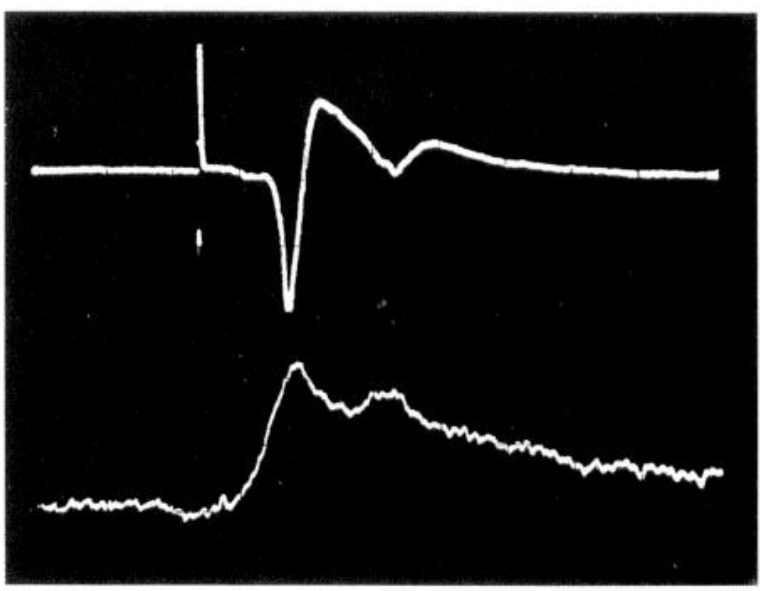

B
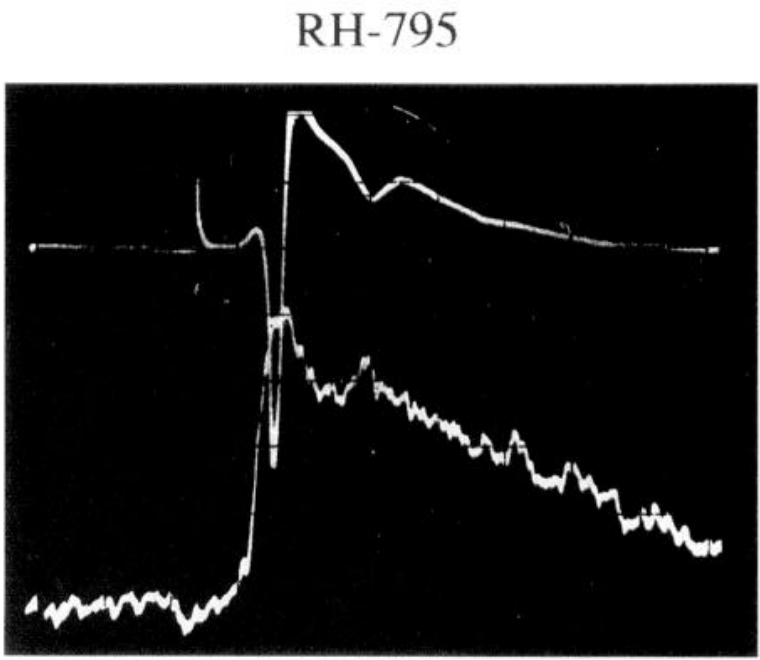

$2 \mathrm{mV}$

$5 \mathrm{msec}$

Figure 4. Comparison of dye responses and LFPs in tissue slices: simultaneous recordings of field potential, recorded with a glass microelectrode (upper traces), and the optical signal, recorded with a photodiode, from area CA1 in a hippocampal slice (lower traces). $A$, Results obtained using the dye $\mathrm{RH}-773$ (structure in Fig. 18). The fractional change of the optical signal was $3 \times 10^{-3} \cdot B$, Results obtained using the dye RH-795. The fractional change was $4.2 \times 10^{-3}$. Single trials taken directly from the screen of the storage oscilloscope are shown. The optical recordings are DC recordings with auto-zero offset (see Materials and Methods). Note the fast response time of voltage-sensitive dyes. Calibration: $5 \mathrm{msec}, 2 \mathrm{mV}$.

population activity. Such a relationship is by no means the rule; the PSTH should be similar to the former types of recordings only if the output firing pattern of a given single cell closely follows that of the average input to a population of cells. The PSTH emphasizes action potentials, that is, the output of cortical processing. Thus, both the similarities and the differences between the various types of recordings, shown in Figures 2 and 3, are consistent with what is expected from the nature of these recording techniques. Recent optical and field potential recordings in slices have reached similar conclusions (Toyama, 1991; Plenz and Aertsen, 1993).

Since the optical signal measures the overall sum of the changes in 


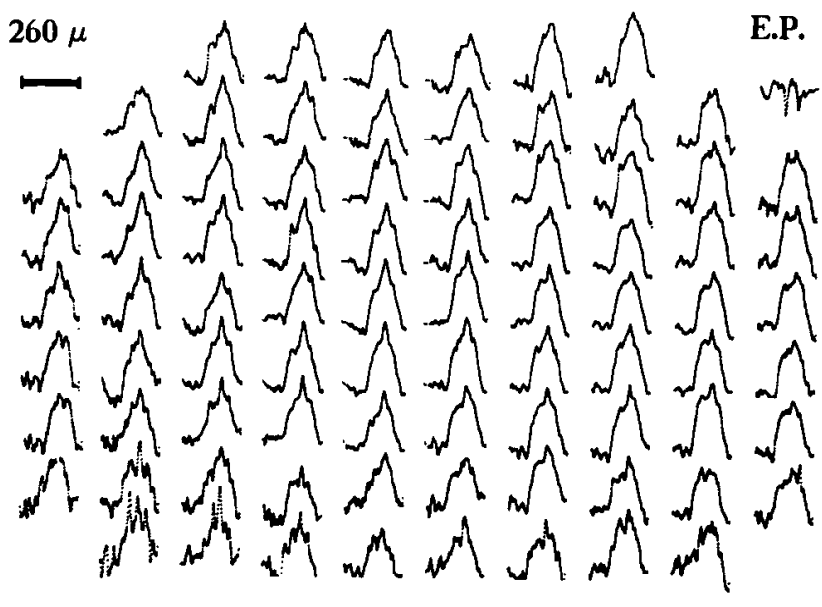

$\mathrm{s}$

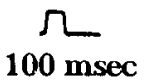

Figure 5. In vivo dye signals in monkey visual cortex: two-dimensional array display of the results from the diode array showing the optical signal, recorded from a 2.6 by $2.6 \mathrm{~mm}$ area of monkey striate cortex. The time course of the signal recorded hy each diode is shown in the corresponding space. The cortex was stained with the dye RH-704 (0.1 $\mathrm{mg} / \mathrm{ml})$ for $1.5 \mathrm{hr}$. The stimulus was a large grating $\left(6^{\circ} \times 4^{\circ}\right)$ of vertical orientation. This stimulus was turned on at times indicated by the bottom left trace. Signal amplitudes were converted to the amplitudes of the corresponding fractional changes in fluorescence by normalization according to the fluorescence intensity detected by each diode. (The noisier signals in the bottom left of the array had a smaller fluorescence level). $S$, stimulus time course; E.P., evoked potential recorded electrically. Calibration pulse (bottom right), $100 \mathrm{msec}$.

membrane potential, a positive optical signal does not mean that inhibition is not present. Inhibition may be masked by a larger excitation and by action potentials in inhibitory axons and nerve terminals. In addition, a large depolarization of the dendrites (i.e., a large optical signal) may not fire a neuron if strong inhibitory synapses hyperpolarize the small cell body or the initial axonal segment. Furthermore, shunting inhibition (without hyperpolarization) cannot be directly detected by voltage measurements. Thus, the inhibitory synaptic potentials (and/or shunting inhibition) are usually masked by the summated EPSP signals. The inhibitory component may be revealed, however, by physiological and pharmacological manipulations as discussed elsewhere (Grinvald et al., 1982b).

Evaluation of voltage-sensitive dyes. The best dye used previously in many preparations was RH-414 (Grinvald et al., 1984), but the dye RH-414 did not stain cat and monkey visual cortices well. It appeared that in these cortices it did not diffuse quickly to stain deep cortical layers. Furthermore, RH-414 affected the microvascular system and led to arterial constriction. Therefore, we have continued our synthesis and dye screening efforts as described in more detail in the Appendix. New styryl dyes were synthesized and then tested on rat hippocampal slices or cat and monkey cortical slices. The dyes were tested for their corresponding response time, optical signal size, photodynamic damage, and pharmacological sidc effects. Figurc 4 shows two examples of dye evaluation in hippocampal slices. In these two cases the size of the evoked field potential before and after the testing was identical. Similar results were obtained from monkey and cat visual cortical slices upon stimulation of the white matter. A comparison between the field potential and optical recordings indicates that the dye response time is in the millisecond range.

Lateral spread of the dye signals evoked by visual stimuli. The 10 best dyes that emerged from the in vitro tissue slice screening (for structure/ performance relationships, see Appendix) were then tested on cat and monkey visual cortex in vivo, using grating of a given orientation as a visual stimulus. Figure 5 shows an example of a screening experiment performed on monkey cortex (M16) with the aid of the diode array. In this experiment the activity was evoked by drifting vertical grating. To a first approximation, activity evoked by gratings of an orthogonal orientation was similar (not shown). Similar results were obtained upon
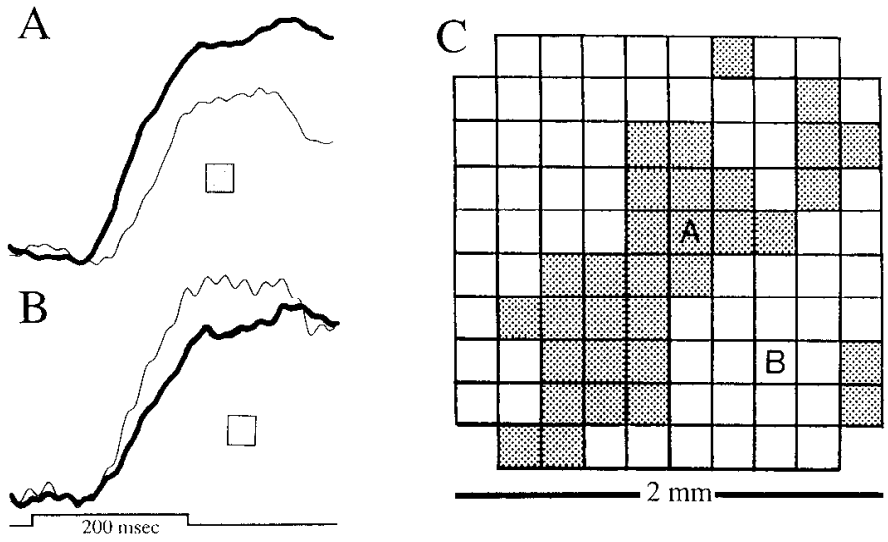

Figure 6. The dependency of dye signals on stimulus orientation. $A$, The amplitudes and time course of signals recorded from one cortical site (marked by an $A$ in $C$ ) in response to two stimuli having orthogonal orientations. Thick lines are responses to vertical gratings. Note that in addition to an amplitude difference there was also a difference in latency between the two responses. (In other loci the latency difference was variable and usually smaller than the one shown here.) $B$, Similar to $A$ but from a cortical patch $\sim 600 \mu \mathrm{m}$ away in which the evoked optical signals exhibited the opposite behavior. Each diode sampled cortical activity from a region of $200 \times 200 \mu \mathrm{m}$. The fractional change in fluorescence, $\Delta F / F$, was $2 \times 10^{4}$. $C$, The shaded areas in the array display show cortical regions in which vertically oriented stimulus produced larger signals than those evoked by an orthogonal stimulus orientation. In the rest of the cortical areas the opposite relationship was observed. The cortex was stained with the dye RH-795. The fractional change was $\sim 3 \times 10^{4}$. Sixty-four trials werc averaged.

testing of several dyes on more than 60 monkeys and cats in this study. The apparently surprising observation, that the signals in the entire fields of 2.6 by $2.6 \mathrm{~mm}$ appear rather uniform, raised the question of why iso-orientation domains were not clearly detected. To address the question of whether a spread of the voltage-sensitive dye signal can account for the apparent blurring of iso-orientation domains, we compared the optical signals obtained with two orthogonal stimuli at each cortical loci. To avoid the complication caused by the responses of "blob" cells, which are not sharply tuned (Livingstone and Hubel, 1984), these tests were also performed in cat visual area 18. Figure 6 illustrates the results of such tests, using two stimuli of orthogonal orientation. To a first approximation the spatial pattern was homogeneous and independent of the stimulus orientation. However, a more careful examination of the dye-related signals as a function of stimulus orientation showed that there were some cortical patches in which the response to a vertical stimulus was only $\sim 70 \%$ of the response to the orthogonal stimulus (e.g., Fig. $6 \mathrm{~A}$; gray regions in Fig. $6 \mathrm{C}$ ). In some other cortical patches the opposite result was obtained as a function of stimulus orientation (e.g., Fig. 6B; white regions in Fig. 6C). We suspected that the differential orientation map (Blasdel and Salama, 1986) obtained by the comparison of the responses to two orthogonal stimuli (Fig. 6C) was related to the spatial distribution of iso-orientation domains. To test this hypothesis, we mapped the iso-orientation domains in the same cortical patch, using an alternative imaging technique based on intrinsic signals (Grinvald et al., 1986). The map obtained by intrinsic imaging was essentially identical. More recently this type of experiment was repeated with much higher spatial resolution utilizing a fast $(2 \mathrm{kHz})$ Reticon array with 4096 pixels (Adaptive Optics) instead of the 100 pixels. Differential maps of orientation domains, obtained by highresolution voltage-sensitive dye imaging and by using intrinsic signals, were essentially identical (D. Shoham and A. Grinvald, unpublished observations).

The relatively uniform distribution of the signals as a function of orientation depicted in Figure 6 was very different from the one expected from single-unit recordings and therefore it deserves additional examination. An amplitude of $70 \%$ for the response cannot be exclusively attributed to light scattering from $500 \mu \mathrm{m}$ away and/or "out of focus" contributions (see below). Assuming an exponential law for the spread from two columns of orthogonal orientation, we calculated that a $70 \%$ reduction of the response over a distance of $500 \mu \mathrm{m}$ corresponds to a 
spread exhibiting a space constant of approximately $1200 \mu \mathrm{m}$. Since we detected such a large spread using a resolution of 200 by $200 \mu \mathrm{m}$ and since the spread detected in the present study fell in the range of 1.5-3 $\mathrm{mm}$, it is clear that a resolution of $600 \mu \mathrm{m} /$ diode used thereafter was sufficient to measure the large spread of the optical signal in monkey cortex.

Photodynamic damage. The duration of optical imaging experiments employing the voltage-sensitive dyes that are currently available is limited by the photodynamic damage (Cohen et al., 1974; Ross et al., 1977) that they cause. For this reason several steps were taken to reduce photodynamic damage. First, we improved the fluorescence collection efficiency of the imaging system by a factor of 20 . Second, a shutter was used to illuminate the cortex only for the duration of the actual measurement. This improvement was facilitated by performing DC measurements here, rather than the $\mathrm{AC}$ measurements used previously; during the previous $\mathrm{AC}$ measurements, evoked signals could not be recorded during the first $1-3 \mathrm{sec}$ of illumination because of the very large transients originating from the AC coupling (Orbach et al., 1985). The modified bank of amplifiers used in the present study contained a sample-and-hold circuit. The net duration of cortical illumination was only 200-500 msec; thus, we cut the duration of cortical illumination by at least fivefold. Third, we evaluated the overall noise of the optical measurement and found that the dominant noise in the experiment was of biological origin. This noise originated from the remaining small irreproducibility in the shape of the heartbeat signal and also from ongoing cortical activity. Ongoing cortical activity is reflected by relatively large optical signals, not related to the stimulus (Arieli, Shoham, and Grinvald, unpublished observations). To minimize this biological noise we reduced the illumination level by a factor of 10 (close to the point where the light shot noise level was similar to the level of biological noise) and thus could perform additional signal averaging to reduce this biological noise.

Taken together, these improvements reduced photodynamic damage by almost three orders of magnitude. As a result, we were able to monitor responses evoked by 10-20 different stimuli, each averaged $20-40$ times, before cortical activity began to degrade as a consequence of photodynamic damage. There are also other factors that could limit the duration of the dye imaging experiments. Accumulation of dye inside of neurons, or delayed pharmacological side effects of the dye, limited the duration of the present experiments to a period of 3-6 hr after the staining (obviously, without continuous illumination).

Pharmacological side effects. The potential pharmacological side effects of RH-795 were tested in vitro using hippocampal slices, and in vivo using single-unit recordings in cat and monkey visual cortex. During in vitro testing the field potential did not change after the staining. In the in vivo tests receptive field properties such as orientation tuning of the units that we studied appeared normal. Thus, we concluded that pharmacological side effects were negligible under the present experimental conditions. However, overstaining of cortical slices in vitro or of cortex in vivo often did produce sluggish responses, and was therefore avoided by using the relatively low concentration of the dye for the staining (the extent of staining was assessed by visual inspection only; dark red/brown color suggested overstaining).

Is the dye signal contaminated by activity-dependent intrinsic signals? We define intrinsic signals as optical signals originating from activitydependent changes in cortical optical properties such as fluorescence or absorption rather than extrinsic probes added to stain the cortex. The most prominent intrinsic signals in cortical tissue originate from changes in the oxidation state of hemoglobin, blood volume changes, and light scattering changes. Any cxtrinsic fluorescent probe should pick up a component of these intrinsic signals, whenever the latter exist, because the intrinsic signals correspond to changes in absorption of excitation light. A change in the intensity of the excitation light surely affects the fluorescence intensity. A common misunderstanding is the belief that if proper filters are used to block the reflected light used for fluorescence excitation, then the activity-dependent fluorescence signals cannot include a component of activity-dependent intrinsic signals. This is not the case, however. Indeed, it has been experimentally demonstrated that activity-dependent intrinsic changes do give rise to a fluorescence signal, even from fluorescent dyes that are not voltage sensitive (Grinvald et al., 1986; Frostig et al., 1990). It has also been shown that the activitydependent intrinsic signal in cat and monkey visual cortex has an onset latency of approximately $250-350 \mathrm{msec}$, including $\sim 100 \mathrm{msec}$ retinal delay of cortical response, and a slow rise time of about $2000 \mathrm{msec}$. Therefore, the short measurements of $250-400 \mathrm{msec}$ performed here can contain only a small contribution of the intrinsic signal. Hence, intrinsic signals do not influence any of the results reported here.

Can a spread of the optical signal be accounted for by out of focus blurring and light scattering? It was necessary to consider the possibility that the spread in optical activity, beyond the retinotopic prediction, was due to out of focus blurring of fluorescence emitted from deeper cortical layers. Also, light scattering of the fluorescence emitted from deep layers could cause the image to smear, even if it were in focus, and this could appear as a spread in optical activity. These topics were previously investigated by Salzberg et al. (1977) and by Orbach and Cohen (1983). The latter reported that light scattering, caused by placing $500-\mu \mathrm{m}$-thick cortical tissue above the focus plane, would blur the optical image by less than $200 \mu \mathrm{m}$; that is, the diameter of the spread (at half-height) was $200 \mu \mathrm{m}$, a "space constant" of $150 \mu \mathrm{m}$ (using a numerical aperture of 0.4). A similar image blur was obtained by defocusing an image by $500 \mu \mathrm{m}$, without the presence of a cortical slice. We performed preliminary experiments in monkey cortex and obtained similar results with the macroscope used here (numerical aperture of 0.4 , also). Thus, spread of optical signal detected in the present study, exhibiting a space constant much larger than $150 \mu \mathrm{m}$, cannot be accounted for by out of focus blurring or fluorescence light scattering.

\section{Results}

Detection of lateral spread of activity beyond the retinotopic activation. To measure the lateral spread of retinotopically restricted stimuli, in the first experiment we used a patch of a drifting grating, which activated the center of the imaged cortical area. The size of this stimulus was $1^{\circ}$ by $1^{\circ}$, and the total imaged area measured $6 \times 6 \mathrm{~mm}$. Thus the diagonal of this area was $8.5 \mathrm{~mm}$. To determine the position of the stimulus, the receptive fields of neurons at the center of the imaged area were mapped with the aid of single-unit recordings (stimulus centered on $6.6^{\circ}$ bclow the horizontal meridian and $2^{\circ}$ away from the vertical meridian). Optical imaging of cortical responses to this stimulus was then performed. The responses to this moving grating were subtracted from the responses to the same grating held stationary

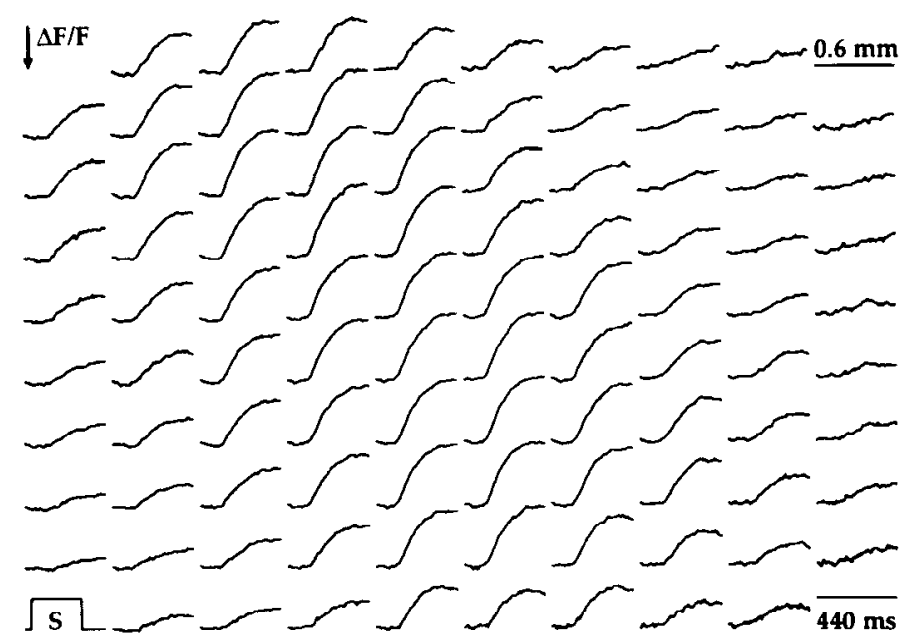

Figure 7. Array display of the optically recorded evoked responses: activity in the left hemisphere of the striate cortex evoked by a small stimulus $\left(1^{\circ} \times 1^{\circ}\right.$ gratings $)$. The time course of each diode is shown in the corresponding space. Each detector viewed an area of $600 \times 600$ $\mu \mathrm{m}$. The total cortical area monitored was $6 \times 6 \mathrm{~mm}$. The timing of the visual stimulus, the fractional change in fluorescence (arrow amplitude $5 \times 10^{-4}$ ), and the time scale are shown at the comers of the array display. The cortex was stained with the dye RH-795 in this and all the following experiments. Twenty trials were averaged. For each trace, the response to drifting grating was subtracted from the "no response" to standing grating of the same orientation (see Results). This type of display of raw data is useful in assessing the quality of the data, but a movie display is far more informative. The macroscope was used in this and all subsequent experiments. 


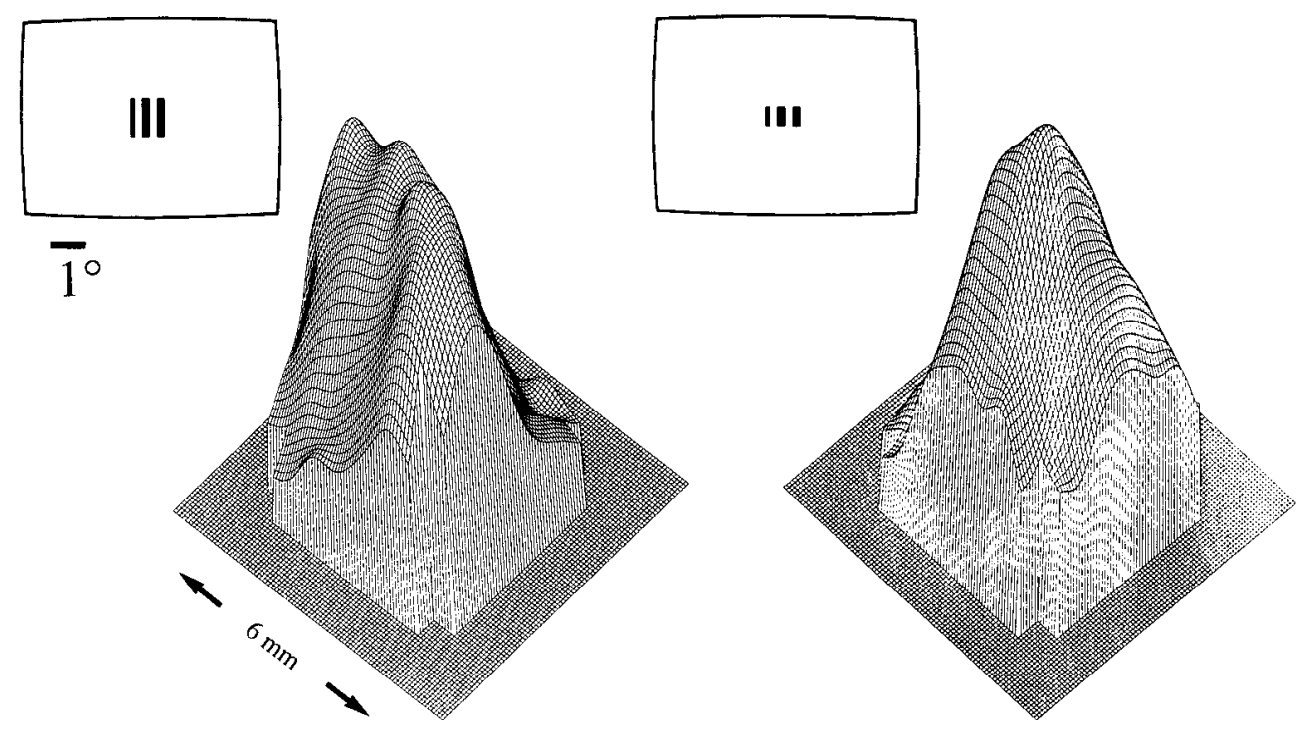

Figure 8. Spatial distribution of cortical activation. Top left, A surface plot display of activity in the left hemisphere of the striate cortex (same as Fig. 7), showing the spatial distribution of the activity $250 \mathrm{msec}$ after stimulus onset. Cortical activity was evoked by a small $\left(1^{\circ} \times 1^{\circ}\right)$ drifting grating shown at the top left. Top right. Surface plot of the activation area in another monkey, evoked by a rectangular drifting grating $\left(1^{\circ} \times 0.5^{\circ}\right), 250 \mathrm{msec}$ after the onset of the drifting gratings. At an early time responses were detected only in a small cortical area near the center. Later, as seen here, the signal spread horizontally and the whole imaged area $(6$ $\times 6 \mathrm{~mm}$ ) showed cortical activity. Bottom. The contour maps of the cortical activation show the asymmetric activation area (including spread) along different cortical axes. Left, Part of the contour map showing the activation area produced by a square drifting grating $\left(1^{\circ}\right), 250 \mathrm{msec}$ after the onset of the drifting gratings. Right, The activation
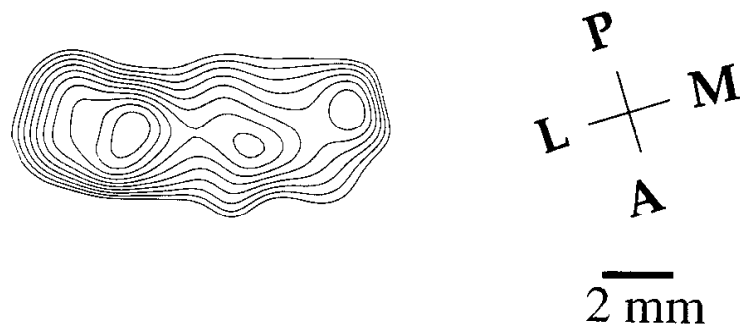
area evoked by a rectangular drifting

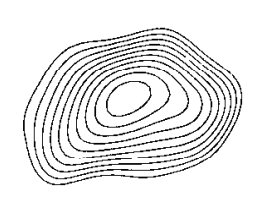
grating $\left(1^{\circ} \times 0.5^{\circ}\right), 250 \mathrm{msec}$ after the onset of the drifting gratings. Note the elongated shape of the response to a square stimulus (left) and the nearly symmetric area of activation in response to an elongated $(2: 1)$ rectangle. $A$, anterior; $L$, lateral; $M$, medial; $P$, posterior. (see Materials and Methods). Figure 7 shows the time course of the evoked cortical activity for all photodetectors. These responses appeared to be somewhat slower than those we had previously observed with flashing drifting gratings (Figs. 2, 5). Figure 8 (top left), taken from an experiment on monkey M26, illustrates a new method of displaying the data (Lieke et al., 1988). A surface plot of the amplitudes of the optically detected signals at each cortical site, $250 \mathrm{msec}$ after the onset of the stimulus, is shown. Similar surface plots were calculated for each time interval with a resolution of $1 \mathrm{msec}$. A contour, topographic map of this surface plot is shown at the bottom of Figure 8. Evidently, the optically detected activity was largest in the center of the cortical area that was imaged, and decreased toward the periphery. While the center of the optically detected activity coincided with the center of the activity detected electrically, the optically detected activity appeared to cover a much larger area than that predicted from previous retinotopic maps for this retinal eccentricity.

To estimate the magnification factor (Daniel and Whitteridge, 1961) of this cortical region (monkey M26) we used four perpendicular electrode penetrations along two different cortical axes. The first pair of penetrations, $10.9 \mathrm{~mm}$ apart, was along an axis nearly parallel to the $\mathrm{V} 1 / \mathrm{V} 2$ border and the second pair, $8.4 \mathrm{~mm}$ apart, was along an orthogonal axis roughly corresponding to the medial-lateral axis. These penetrations were made near the edges of the optically imaged area. The receptive field sizes were $0.3-0.5^{\circ}$ wide, and receptive field scatter (three or four units recorded in each penetration) was only $\sim 9 \%$ relative to the distances between the aggregate receptive fields corresponding to each of the four penetrations. Thus, errors in es- timating the local magnification factors are small. We found that the magnification factor was $3.4 \times$ parallel to the vertical meridian and $1.8 \times$ along the orthogonal axis. Thus, the ratio of the magnification factors along the two axes was 1.9 . We refer to this ratio as the local anisotropy factor.

We used multiple perpendicular penetrations to prove that the relatively large spread of optical signal included cortical regions in which most single units did not respond to the small gratings stimulus. This experiment included 16 penetrations, in each of which we recorded from at least two neurons, and was performed on another monkey (M27) at the end of the optical imaging session. We again found that single units responded to the gratings only in a small cortical region. A dozen of these recordings were aimed along the borders of the optically imaged area where relatively large optical signals were still observed. First, at the same 12 sites we found active single or multiple units using a hand-held projector and noted that the receptive fields of these units were outside the area occupied by the small gratings. We then tried to activate these units with the small gratings. However, we did not detect any clear unit activity in response to presentations of the gratings at any orientation. Thus, a comparison of the results from these electrical recordings to the bell-shaped activation regions detected optically (Fig. 8 ) indicated that the optical signals spread beyond the regions where single-unit activity could be readily detected.

Anisotropy of the optically detected cortical activation. To determine whether the optically detected activation area was anisotropic, as previously shown for this cortical region (Van Essen et al., 1984; Dow et al., 1985; Tootell et al., 1988), we inspected the contour topographic maps obtained from the optical imaging 


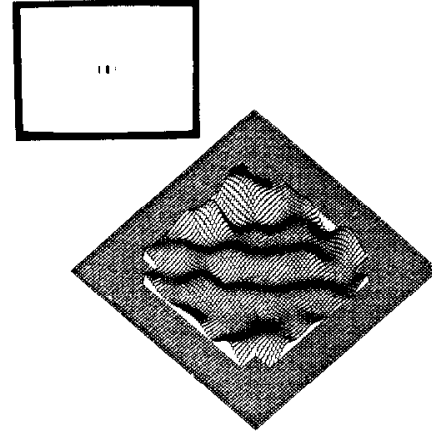

$0 \mathrm{msec}$
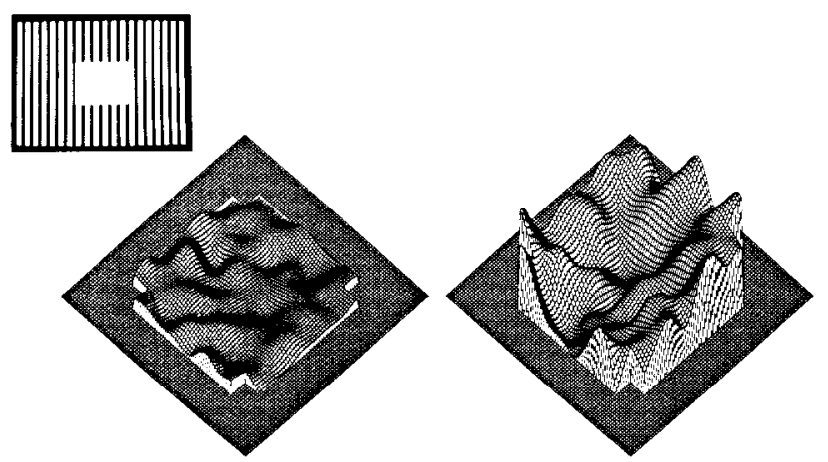

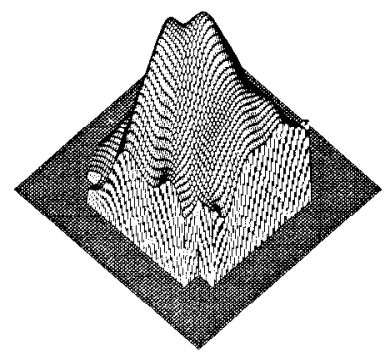

$200 \mathrm{msec}$

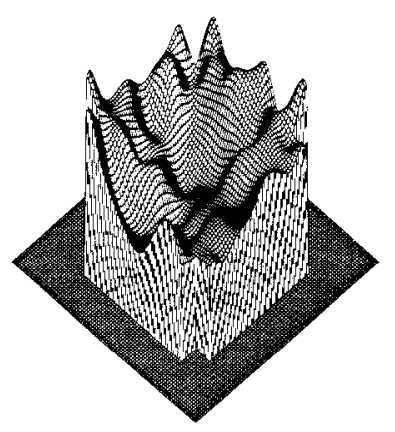

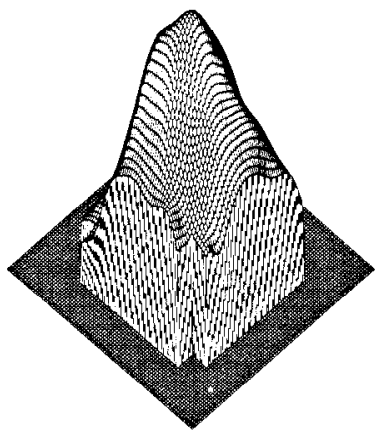

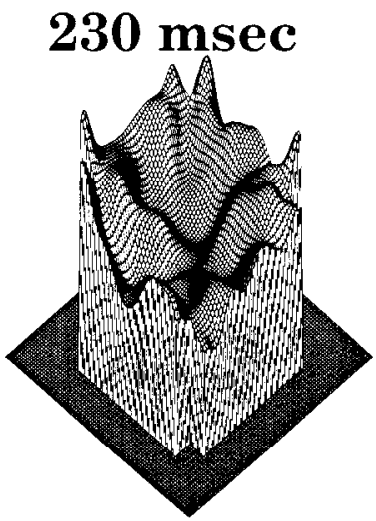

Figure 9. Frames from a movie showing the lateral spread of the activity. The two sequences of four frames, out of a total of 250 frames, were taken at the beginning of the experiment, $160 \mathrm{msec}, 200 \mathrm{msec}$, and $230 \mathrm{msec}$ after stimulus onset. The initial response could be detected $\sim 100$ insec (mostly retinal delay) after stimulus onset. Top, Lateral spread of cortical activation produced by a $1^{\circ} \times 0.5^{\circ}$ small drifting grating, ccntcred for the activation of this $6 \times 6 \mathrm{~mm}$ patch of cortex. The activated area was much larger than expected from direct retinotopic activation. Bottom, Four frames showing the cortical activation in response to a large surround stimulus with a $3^{\circ} \times 2^{\circ}$ mask (hole) at its center. This mask prevented direct activation of nearly the entire imaged patch of cortex, as we confirmed with single-unit recordings. Note, however, that the activity spreads from the periphery to the center of the imaged area. The first frame in each sequence shows the "noise" level associated with the present experiment. Twenty trials were averaged.

data. Figure 8 illustrates two contour maps obtaincd from two monkeys. In one experiment (from M26), when a $1^{\circ} \times 1^{\circ}$ square grating was used as a visual stimulus, the cortical activity covered a rectangle-like area rather than a square (left map). The long axis was approximately parallel to the V1/V2 border. The ratio of the long axis to the short axis was 2.4. The anisotropy factors calculated from the optical data included both the directly activated area and the regions of spread. This anisotropy factor of 2.4 obtained from the optical imaging should be compared with the value of 1.9 measured by single-unit recordings. This discrepancy will be discussed later. In a second experiment, performed on another monkey (M27) but at an eccentricity of $8^{\circ}$, the size of the visual stimulus was $1^{\circ} \times 0.5^{\circ}$. The result is shown in the right side of Figure 8. Following the correction for the aspect ratio of this stimulus (2.0), a similar anisotropy factor, 2.6, was obtained in this experiment. Thus, the evoked optical signal was highly anisotropic at these cortical locations, close to the $\mathrm{V} 1 / \mathrm{V} 2$ border, confirming previous reports using traditional techniques.

"Topographic maps" of optically detected activity, of the type shown in Figure 8, contain fine details of peaks and valleys. Many of these were reproducible in multiple imaging experiments from the same cortical regions. Most prominent are the three peaks shown in Figure 8 .

Cortical activation produced by center and surround stimuli. A second way to demonstrate the long-range spread of visually evoked activity was to stimulate cortical regions outside the imaged cortical region by using "surround stimuli." Thus, for the small stimulus used in the previous experiment, we substituted a large grating $\left(6^{\circ} \times 9^{\circ}\right)$ with a $2^{\circ} \times 3^{\circ}$ mask ("hole") in its center. The sequence of events from the onset of these two types of stimuli is best seen by inspecting a movie in slow motion. The movie is composed of many surface plots, each of which is a movie frame, obtained by real-time optical imaging. Figure 9, taken from an experiment on monkey M27, shows four frames from the movie for both a small "center" stimulus and a "surround" stimulus. In each case, the activity spreads far away from its point of initiation, either from the center toward the periphery (for the small center stimulus) or from the periphery toward the center (for the surround stimulus). Quantitative results will be presented after the next section.

Time course of the spread of electrical activity. To compare the timing of cortical activity evoked at the center with that at the edge of the imaged cortical field, we plotted the time course of central and peripheral detectors (Fig. 10A). The normalized slopes of the responses in the various cortical loci were similar, but the peripheral response was delayed by approximately 40 msec relative to the center response (Fig. 10B). This time difference corresponds to an apparent conduction velocity of 0.11 $\mathrm{m} / \mathrm{sec}$ for the horizontal spread of activity. Complementary results were observed using the surround stimulus (Гig. 11). Again, the time course for the spread of cortical activity was similar to that observed in the previous experiment, but with the surround stimulus the timing relationship was reversed. That is, 
A

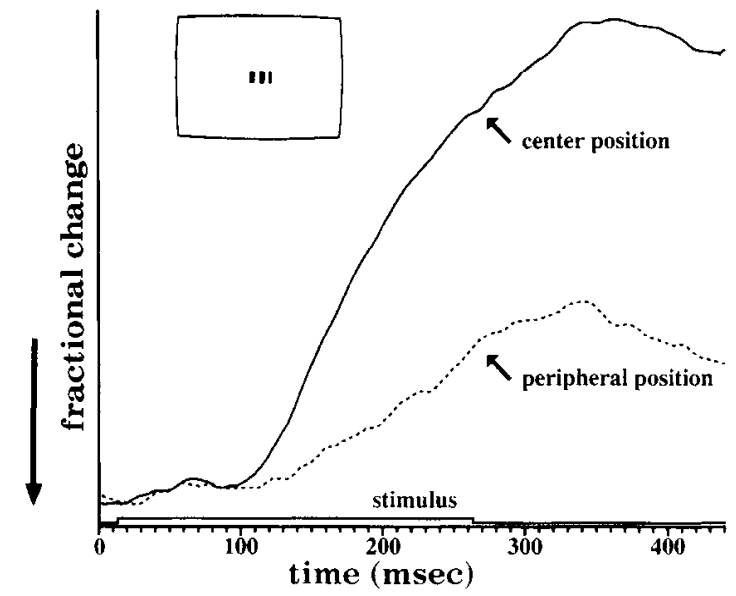

$\mathrm{B}$

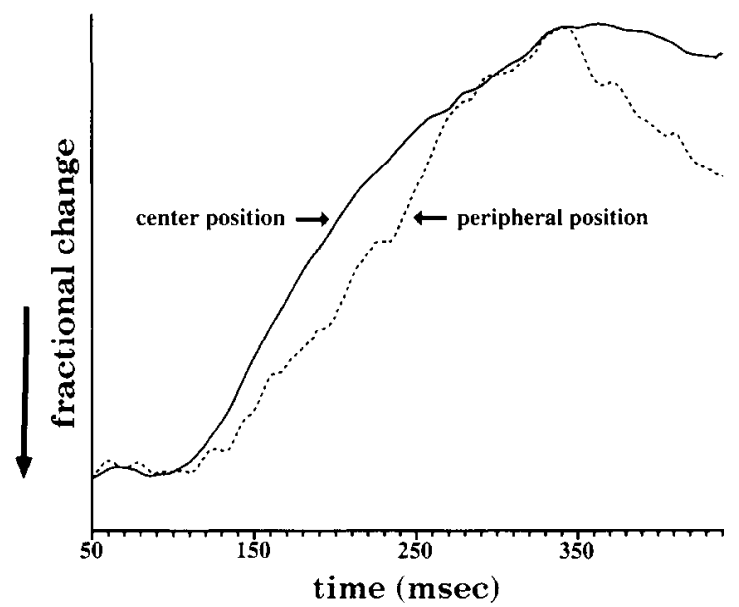

Figure 10. The time course of optical signals at different cortical sites and estimation of the spread velocity. Cortical activity was elicited by a $1^{\circ}$ $\times 0.5^{\circ}$ small moving grating ("center-only" stimulus). $A$, Optical signals from the center (continuous line) of the imaged cortical area and the periphery, $\sim 4.5 \mathrm{~mm}$ away (dashed line), are shown. $B$, The amplitudes of signals recorded from the center (direct activation) and the periphery (indirect activation by spread) were normalized to emphasize the difference in response latency and in rise times. The latency difference of about $40 \mathrm{msec}$ was estimated by comparing the time to half-height (arrows) of each signal, measured about $4.5 \mathrm{~mm}$ away from each other.

the response in the center of the cortical patch was delayed relative to that in the periphery. The speed of signal spread was $0.24 \mathrm{~m} / \mathrm{sec}$. We calculated the speed (apparent conduction velocity) from eight measurements on monkeys M08 and M24$\mathrm{M} 27$, and found it to span the range of $0.09-0.25 \mathrm{~m} / \mathrm{sec}$. This conduction velocity could be mediated by various cortical mechanisms. It is consistent with cortical activity spreading laterally and being directly mediated by long-range, nonmyelinated connections. A second alternative is that the spread is mediated by polysynaptic pathways, within the same cortical regions mediated by intrinsic myelinated axons exhibiting faster conduction. A third alternative consistent with the data is that the spread is mediated by feedback from higher cortical areas and myelinated axons. After all, at least nine higher visual areas feed back into V1 (Felleman and Van Essen, 1991). Here we did not distinguish between these three possibilities and/or their various combinations.

It is also surprising that the amplitude of the evoked activity (as monitored optically) continued to increase rather slowly (Figs. 9, 10), whereas PSTHs from most single-unit recordings are known to be either flat or show a moderate decline. Here, 350 msec after the stimulus onset, the amplitude of the response was approximately threefold larger than at $150 \mathrm{msec}$ (Fig. 10).

Cortical "space constant" for the lateral spread and its anisotropy. From the previous figures it is apparent that the optically detected cortical activity does not show sharp borders as a result of stimulation of a retinal area with a stimulus containing sharp borders. What we see instead is a monotonically declining spread that can be approximated by a Gaussian or exponential curve. To determine if the spread of the optical signal itself, beyond the directly activated cortical region, was also anisotropic, we compared the activation patterns produced by center and surround stimuli. If the spread were isotropic one would expect that the contour plots of the mountain-like and the craterlike activation would have nearly the same aspect ratio. However, if the spread were significantly anisotropic, then the aspect ratio for the corresponding contours would be very different. Figure $12 A$, taken from monkey M27, shows the contour map (bottom right) of the mountain-like activation area produced by the center stimuli of $0.5^{\circ} \times 1^{\circ}$. The longer axis of the ellipsoid contours was along the lateral medial axis, parallel to the V1/ V2 border. Figure $12 B$, taken for an interlaced measurement, shows the crater-like activation area produced by the $2^{\circ} \times 3^{\circ}$ surround stimulus (bottom right). In this case, however, the longer axis of the crater's contours was found along the anteriorposterior axis. One must conclude, therefore, that the spread of the optical signal along the orthogonal axis was significantly larger. Thus, at this eccentricity the spread parallel to the V1/ V2 border, known to be perpendicular to ocular dominance columns, was larger than along the ocular dominance columns. The same result was obtained upon inspection of the corresponding contour plots of monkey M26.

To quantify the extent of the spread of activity, we measured

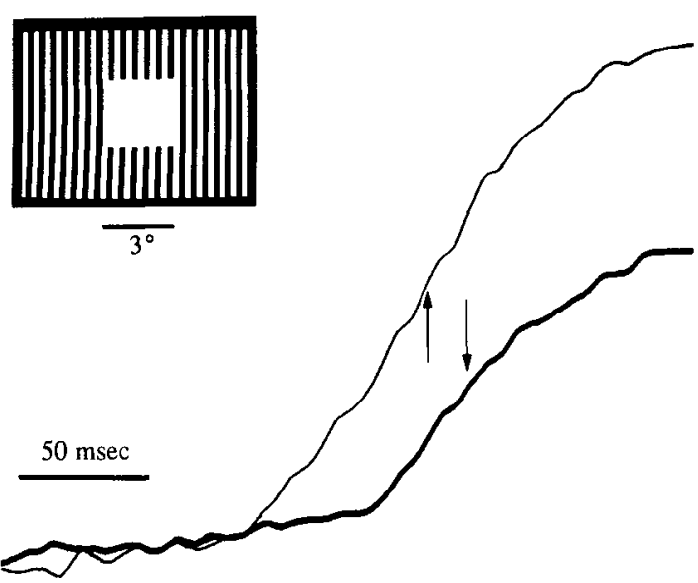

Figure 11. Spread from the surround to the center. Optical signals elicited by a large moving grating with a mask (hole) at the center ("surround" stimulus). The mask was $3^{\circ} \times 3^{\circ}$, so that cells retinotopically activated by the surround stimulus were at least 2.7 to $5.1 \mathrm{~mm}$ away from the center of the imaged area. The thick line trace is from the center of the imaged area (indirect activation) and the thin line trace is from the periphery. The latency difference of about $15 \mathrm{msec}$ was measured by comparing the time to half-height of each signal (arrows). The two cortical sites were $3.6 \mathrm{~mm}$ away from each other. 


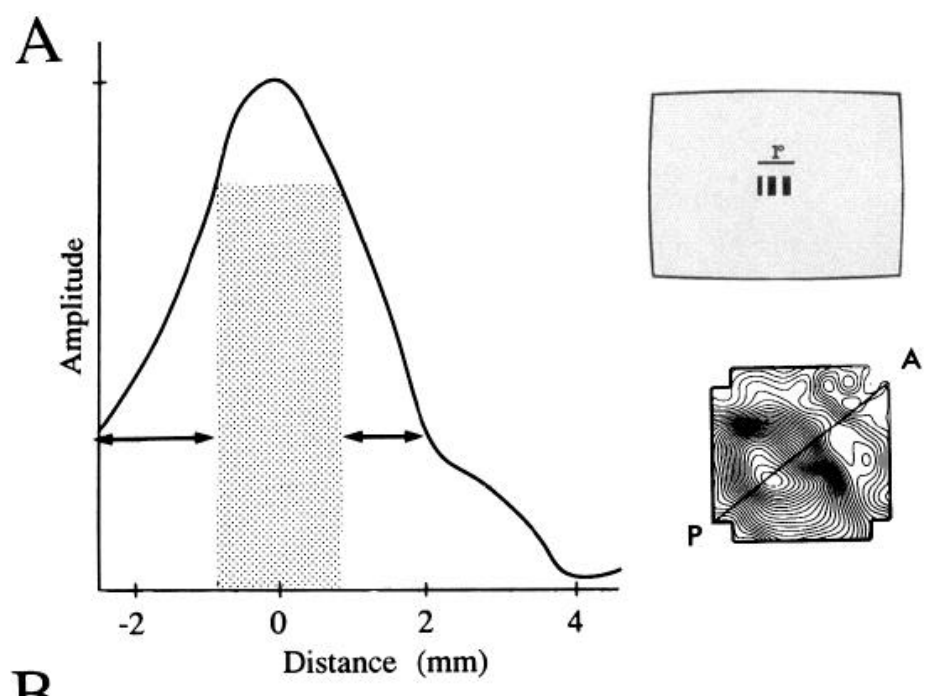

B

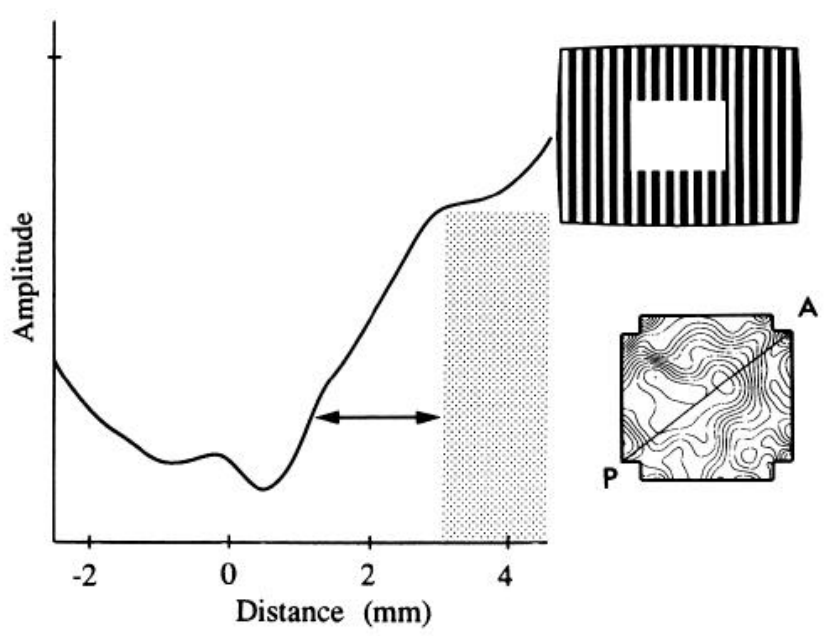

Figure 12. The "space constant" of the lateral spread of the optical signal. $A$, The amplitude of the evoked signals as a function of cortical distance. The stimulus is shown at the top right $\left(1^{\circ} \times 0.5^{\circ}\right.$ drifting grating). The bottom right shows the contour map for the pattern of cortical activation, $350 \mathrm{msec}$ after stimulus onset. The amplitude of the evoked cortical activity plotted at the left was measured along the diagonal line in the contour plot shown at the bottom right. The shaded area corresponds to the area of direct activation, predicted from the magnification factor. The "space constant" calculated along a second diagonal line, orthogonal to that shown in the contour plot, was about two times larger than that shown here. For bell-shaped curves like the one shown here, the calculated values of the "space constants" are not sensitive to an error in the size of the area of direct activation. $B$, The lateral spread evoked by a surround stimulus. Left, The amplitude of the evoked signals as a function of cortical distance, measured along the diagonal line shown in the contour plot at the bottom right. The stimulus (large grating with a $3^{\circ} \times 2^{\circ}$ mask at the center) is shown at the top right. The bottom shows the contour map for the pattern of cortical activation, $350 \mathrm{msec}$ after stimulus onset. The shaded area corresponds to the area of direct "surround activation" by the edge of the peripheral stimulus, calculated from the magnification factor we measured (see Results).

the amplitude of the optical signal as a function of cortical distance. Figure $12 \mathrm{~A}$ shows the amplitude of the evoked activity, as a function of cortical distance, along the anterior-posterior axis, $350 \mathrm{msec}$ after the onset of a center stimulus. From the magnification factor we estimated that the length of the directly activated region along the anterior-posterior axis chosen for this graph was $\sim 1.8 \mathrm{~mm}$. We define the space constant for the optically detected spread as the cortical distance at which the optical signal declined to 1/eth of its amplitude at the "retinotopic border." A value of $1.5 \pm 0.25 \mathrm{~mm}$ was found (the average length of the two double arrows shown in Fig. 12A). The value of the space constant for the spread along an orthogonal axis, the mediolateral axis, was calculated from the same topographic contours of the optical signals shown in the inset of Figure $12 \mathrm{~A}$, and was found to be approximately twofold larger: $2.7 \pm 0.2$ $\mathrm{mm}$.

Figure $12 B$ shows similar data for the surround stimulus. The amplitude of the evoked activity $350 \mathrm{msec}$ after the onset of the surround stimulus, as a function of distance along the cortical surface, is shown. Again, from the magnification factor we estimated the border position of the directly activated region along the axis chosen for this graph (shaded area, corresponding to the shoulder in the amplitude vs distance curve). The space constant calculated for the spread was $\sim 1.7 \mathrm{~mm}$ (the length of the double arrow in Fig. 12B). Note that because of the bell shape of these curves the values of the space constant estimated here are not very sensitive to the error in estimating the retinotopically activated region. To estimate such an error we calculated from the experiment with M27 what the space constant would be if the directly activated area was $50 \%$ larger. The value of the space constant thus obtained was $1.4 \mathrm{~mm}$ rather than 1.5 $\mathrm{mm}$ obtained above.

In another experiment, another monkey (M25) viewed a surround stimulus with a $6^{\circ}$ by $6^{\circ}$ square hole. Thus, for this stimulus the center of the imaged area was $3^{\circ}$, at least $6-12 \mathrm{~mm}$ away from retinotopically activated borders of the stimulus. The spread of activity at the center of the imaged area was still detected; its amplitude was $5-10 \%$ of that evoked in this area by a small center stimulus.

Eight measurements of the "space constants" for the lateral spread beyond the retinotopic border, from experiments on two monkeys (M26 and M27), showed that the space constant for the spread of the optical signal falls in the range of $1.4-1.8 \mathrm{~mm}$, perpendicular to the V1/V2 border. An approximately twofold larger space constant for the spread along the axis parallel to the $\mathrm{V} 1 / \mathrm{V} 2$ border was observed (eccentricity between $6^{\circ}$ and $8^{\circ}$ ).

Lateral interactions between responses to "center" and "surround" visual stimuli. Since a finding of an optical signal with a positive polarity does not imply that it lacks inhibitory components (see Materials and Methods), our next step was to determine whether the spread of activity had an inhibitory component. To evaluate the functional nature of the lateral spread and its implications for cortical signal processing, we used more complex stimuli, in which both the center and surrounding cortical regions were stimulated simultaneously. Each experiment consisted of four interlaced stimulus conditions: center stimulus alone, surround stimulus alone, both stimuli at the same time and at identical orientations, and both stimuli together but with orthogonal orientations of the center and the surround. A $1^{\circ}$ gap was maintained between the center and the surround stimulus regions.

Surround inhibition. Figure 13 shows surface plot images of the optically detected activity evoked by three different stimuli $\sim 230 \mathrm{msec}$ after onset of the cortical response. The large grating with the "hole" elicited a large response in the periphery of the imaged area, with some spread to the center, producing a "cra- 

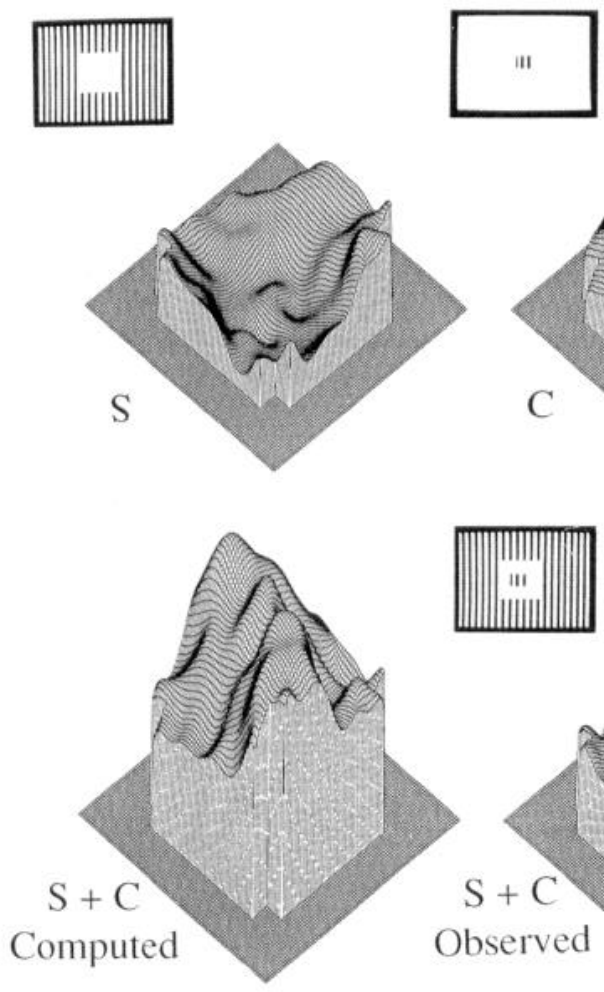

C
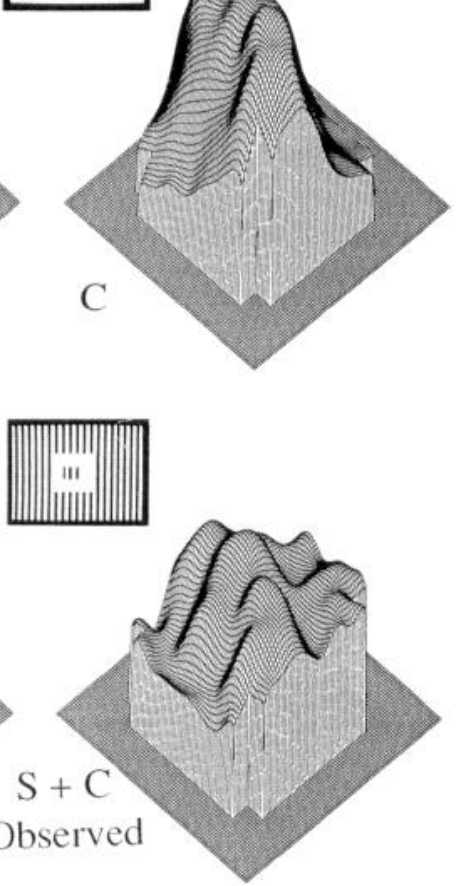

Figure 13. Surface plots illustrating surround "inhibition." Top left, A large vertical grating with a mask at the center produced a "craterlike" spatial response distribution. Top right, A small vertical drifting grating that produced an almost mountain-like distribution. Bottom left, The calculated linear sum of the two responses shown at the top. Bottom right, The actual response pattern observed when the two stimuli were combined. Note the "inhibitory" nature of the interactions; in the center of the image, the response is attenuated by a factor of $0.3-0.4$. The surface plots show the activation patterns $230 \mathrm{msec}$ after onset of the cortical activity. $S$, surround; $C$, center. "Topographic maps" of optically detected activity, of the type shown in Figure 8 and here, contain fine details of peaks and valleys. These are reproducible in multiple imaging experiments from the same cortical regions. The distance between the two extreme peaks was $3.5 \mathrm{~mm}$, which may correspond to objects in visual space separated by $\sim 1.03^{\circ}$. Since the two bars were $1^{\circ}$ apart, the locations of the two peaks nicely correspond to the distance between the two bars of the stimulus. Therefore, we suspect that the three peaks correspond to the three bars of the small grating. One reason why one might expect to see three bars is probably related to the fact that the three-bar stimulus was stationary during the interstimulus interval. Other explanations related to ocular dominance columns were not ruled out.

ter-like" surface plot of the response (top left). The small grating produced a large response in the center of the imaged area with considerable spread toward the periphery (top right). The computed linear combination of "center" and "surround" responses is illustrated in the bottom left. The actual result observed for the combined stimuli is shown at the bottom right, and was very different from the linear combination. The response, at the center of the imaged area, was attenuated by $34 \%$ relative to that evoked by the center stimulus alone. Relative to the computed amplitude of the linear combination of center and surround responses the response was attenuated by $\sim 39 \%$. This suggests that the surround activation had a large suppressive effect on the center activation, and vice versa. With this stimulus configuration, an opposite orientation of the surround stimulus had the same suppressive effect on the activity evoked by the center stimulus (at the center of the imaged area). To test how such attenuation of the signal depends on the distance between the two adjacent stimuli, we varied the distance between the center and surround stimuli, in an experiment performed on a different monkey (M24). When a set of four center plus surround stimuli were interlaced in a single imaging session, the extent of the attenuation of the center signal diminished as the distance between two adjacent stimuli increased. However, a 5-10\% signal attenuation was still observed when the edge of the surround stimuli was $3^{\circ}$ away from the border of the center stimulus.

A cortical origin of iso-orientation inhibition. The next experiment was designed to determine whether the presumed cortical inhibition detected here originated solely in the retina and lateral geniculate body, or whether a portion of it was cortical in origin. Surround inhibition has been detected both in the retina and the lateral geniculate body, stations of the visual system prior to the visual cortex (McIlwain, 1966; Cleland et al., 1971; Kruger and Fischer, 1973; Kaplan and Shapley, 1989). Sharp orientation selectivity is first created in the cortex itself. Therefore, if different inhibitory effects are observed using two surround stimuli that differ only in their orientation, this would indicate that at least part of the inhibition is of cortical origin, rather than of retinal or geniculate origin alone. Thus, one way to assess the contribution of a cortical component to the surround inhibition is to demonstrate that the surround inhibition is orientation dependent. As already mentioned, when we interlaced two experiments similar to those described in Figure 13, but in which the surround stimuli had orientations orthogonal to the center stimulus, no significant difference in amplitudes of the response to the center stimulus was observed. Since the interactions may also depend on the spatial parameters of the stimuli, we decided to vary the shape and location of the surround stimulus.

We reduced the size of the surround stimulus and positioned two small gratings only along the two sides of the center stimulus. This provided a more localized surround stimulus, one that did not extend along the long axis of the bars in the center stimulus. The results in Figure 14 show the surface plots of the responses for the two combined stimuli, $250 \mathrm{msec}$ after onset of the response. The difference between the response pattern produced by the center plus an "orthogonal" surround and that produced by the center plus a "parallel" surround is shown at the bottom right of Figure 14. Evidently, using this configuration of stimuli, the "parallel" surround generated a stronger attenuation than the "orthogonal" surround. The same result was obtained in two independent measurements carried out on the same cortical patch.

The spatial nature of the interaction deserves further examination. We subtracted frames obtained using center and orthogonal surround stimuli from corresponding frames obtained using center and parallel surround stimuli (Fig. 14, bottom right). To demonstrate that the observed differential pattern is significant, we show the noise level associated with such measurements (Fig. 14, bottom left). Here, similar to the above procedure, frames from each of the two measurements were subtracted, but those frames were obtained before the onset of the response to the stimulus. The baseline noise was rather flat, as we noticed upon inspection of a movie composed of 30 subtracted frames. An example of the baseline noise is depicted by the frame labeled "base line" shown in Figure 14. Since the peak difference that was observed for the spatial pattern depicting the orientationdependent interactions was three- to eightfold larger than the 

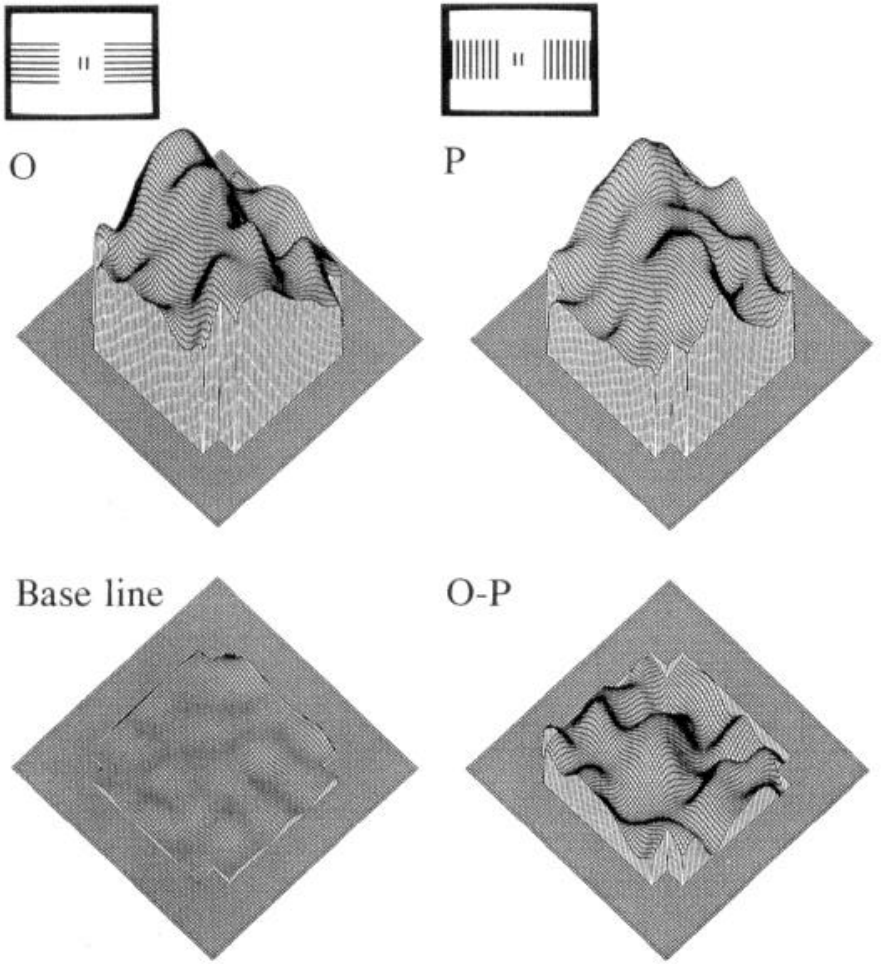

Figure 14. Center surround iso-orientation suppression: surface plots $250 \mathrm{msec}$ after response onset. Top left, The combined center and surround stimuli had orthogonal orientations $(O)$. Top right, The surround stimuli were changed to the same orientation as that of the center $(P)$. The bottom left surface plot shows the noise level in the experiment (see Results). The bottom right shows the significant difference between the two images at the top, suggesting that iso-orientation suppression was larger than the suppression produced by orthogonal stimuli. Since we did not compare the activation pattern evoked by two surround stimuli of opposite orientations alone, one may wonder if uncorrected astigmatism could account for the difference in the effect of the two stimuli. However, in a previous measurement on the same cortical area (Fig. 13), the two surround stimuli with different orientations did produce the same level of suppression of the activity evoked at the center, suggesting that both orientations were effective. Another puzzling aspect is the shape of shoulders and valleys all around the periphery of the differential image $O-P$. Most of these cortical regions were not directly activated by the "center" or surround stimuli. From what is known on $\mathrm{V} 1$, one may expect some fluctuations in the spatial pattern that should be related to orientation domains around the mean level of activity. With the low spatial resolution used here aliasing could occur. Therefore, this part of the pattern exhibiting lower amplitude relative to that at the center of the image should not be overinterpreted. Edge effects and illusionary contours may further contribute to the pattern at the periphery of the imaged area.

noise level, we conclude that much of the observed spatial pattern for the difference is significant (O-P, bottom right).

The histogram shown in Figure 15 illustrates the average amplitudes of the responses of eight neighboring detectors that covered the central region of the cortical image. The signal attenuation ranged from $11 \%$ to $23 \%$ depending on the relative orientation of center and surround stimuli. Evidently, the same orientation produced about twice as much attenuation $(23 \%)$ as that produced by the stimulus having orthogonal orientations $(11 \%)$. This result was reproduced in three measurements (mean, $21 \pm 6 \%$ and $14 \pm 5 \%$, for parallel and orthogonal orientations, respectively). Thus, for the stimulus parameters and spatial relationships used here, these results are consistent with the interpretation that suppression is larger when the surround stim-

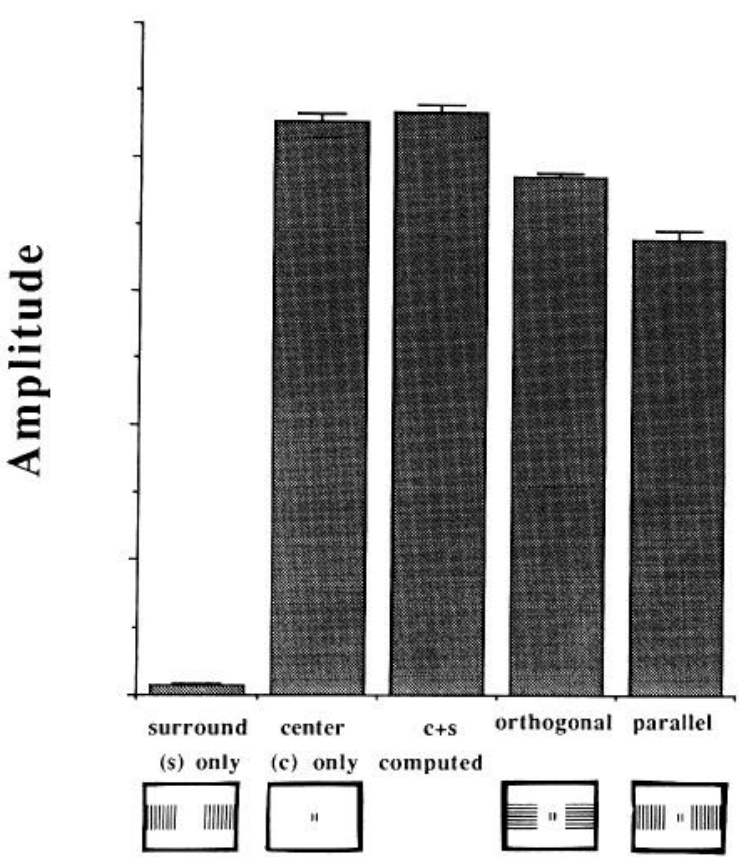

Figure 15. Histogram showing the effect of the orientation of the surround stimuli. The center stimulus was a vertical drifting grating $\left(1^{\circ} \times\right.$ $\left.1^{\circ}\right)$. Surround stimuli of either vertical or horizontal orientation were positioned along the two sides of the center grating at a distance of $1^{\circ}$. The histogram bars show the average amplitude of the responses for each stimulus condition from eight neighboring detectors that monitored activity at the center of the imaged cortical area (about $200 \mathrm{msec}$ after response onset). The error bars display the SD for an average over $20 \mathrm{msec}$. The differences between the center stimulus (second bar from left) and the combined stimuli (bars 4 and 5) are all highly significant $(p<0.001)$.

ulus and the center stimulus have the same orientation, at the level of a large population of neurons.

The relation between suppression and the size of the surround stimulus. The next set of experiments was designed to provide additional evidence for the cortical origin of the suppression. If the suppression was of retinal or geniculate origin one would expect that a large surround stimulus would produce larger suppression relative to that of a smaller surround stimulus. As discussed below, the opposite may be true for inhibitory interactions of cortical origin. We therefore designed a set of sizedependent stimuli in order to assess the cortical contribution to the inhibitory effect.

The surround stimulus had two gratings positioned above and below the center stimulus. The orientation of the surround grating was kept parallel to that of the center stimulus, and they drifted in opposite directions. For the first stimulus we used short bars for the surround gratings (length of $0.5^{\circ}$ ). For the second stimulus we used long bars for the surround stimulus (length of $1.5^{\circ}$ ). Two other stimulus conditions were interlaced with the above stimuli, a center stimulus alone and a surround stimulus. The responses to the center and surround stimuli alone were similar to those obtained in the previous experiment shown in Figure 13. In addition, the combined stimuli showed, once again, attenuation of the responses at the center of the imaged cortical area. The histogram of Figure 16 shows that the attenuation of the center response was larger when the surround stimuli had short bars. One possible explanation for this result is to attribute it to the effect of end-inhibition, which is thought to originate primarily from intracortical interactions (Bolz and 
Gilbert, 1986). The present finding is consistent with the observation that many cortical cells in $\mathrm{V} 1$ are end-inhibited. Under this interpretation, the short bars in the surround activated the immediate surround better than the long bars, and therefore produced a stronger inhibitory effect on the cortical responses evoked by the adjacent center stimulus.

\section{Discussion}

The first major finding of this study is that local activity in the primary visual cortex quickly spreads far beyond the distance that might be expected based on previous data for the retinotopic organization of layer IV and the physiology of its connections to other cortical layers. $\Lambda$ second finding is that this spread is approximately twofold larger perpendicular to the ocular dominance columns than along the columns. A third, related finding is that the surround stimuli that we employed always suppressed the response to a center stimulus. A fourth finding is that some adjacent stimuli with matched orientations produced greater suppression than that of adjacent stimuli with orthogonal orientations. All of these findings relate to the averaged response of a large population of cells rather than to a sample of single neurons; approximately 60,000 neurons lie under a single photodiode. Below we discuss these findings, along with previously available data, in the context of the new approach that we used to explore the cortical point-spread function in space and time, and the effect of lateral cortical interactions. While these results may appear surprising at first sight, several independent studies actually lend support to the present findings and interpretations. Finally, the disadvantages and merits of real-time optical imaging relative to imaging based on intrinsic signals will be discussed.

The spread of optically detected cortical activity. Using realtime optical imaging and inspecting movies from the experiments reported here we observed that the optical signal, which presumably reflects the activity in the primary visual cortex, spreads quickly and far beyond the retinotopic activation (Hubcl and Wiesel, 1974; Tootell et al., 1982, 1988; Van Essen et al., 1984; Dow et al., 1985); 350 msec after the onset of the stimulus the optical signals covered a cortical region much larger than 6 by $6 \mathrm{~mm}$ (Fig. 9), as opposed to an area of approximately $2 \times$ $4 \mathrm{~mm}$, for the $1 \times 1$ stimulus, predicted from previous measurements at the corresponding eccentricities (e.g., Tootell et al., 1988) and confirmed by our own single-unil recordings. Could the spread detected here be an artifact of the method or of the experimental procedure we used?

One explanation for the observed spread is to assume that it is simply an artifact originating from residual eye movements. However, eye movements were not a problem in our short imaging sessions (see Materials and Methods). Light scattering of the fluorescence and image blur from out of focus layers can also cause some spread of optical signals. As explained in Materials and Methods, these problems cannot account for the large spread detected here. Also, it would be difficult to explain the anisotropy of the spread itself, first observed here, if the spread was merely such an artifact. Furthermore, since the optical signal not only spread laterally but also exhibited a different latency relative to retinotopically evoked activity (Figs. 10,11), out of focus blurring and fluorescence light scattering cannot explain such spread in activity. Another artifact that may produce an apparent spread and latency differences is contamination of the fluorescence signal by slow intrinsic signals originating from nonlocalized oxygen delivery and blood volume changes. As

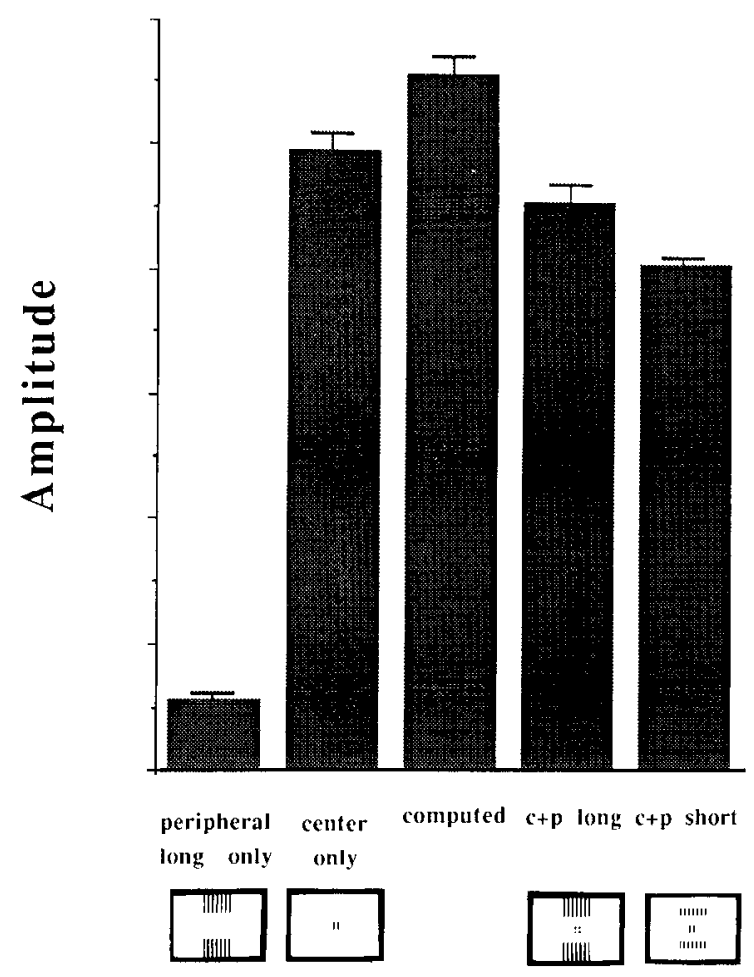

Figure 16. The effect of short and long bars in the surround stimulus on the response at the center. The center stimulus was again a vertical drifting grating $\left(1 \times 1^{\circ}\right)$. Surround stimuli of the same orientation but having different length bars were positioned above and below the center stimulus. The histogram bars show the average amplitude of the responses for each stimulus condition from four neighboring detectors that monitored activity at the center of the imaged cortical area. Long bars $\left(2^{\circ}\right)$ in the surround $(c+p$ long) produced suppression when compared to the computed linear combination of responses to the two stimuli. However, shorter bars $\left(1^{\circ}\right)$ in the surround $(c+p$ short $)$ produced even greater attenuation. The error bars display the $\mathrm{SD}$ for an average over $20 \mathrm{msec}$. The differences between the center stimulus (second bar from left) and the surround stimuli with short and long gratings (bars 4 and 5) are all highly significant $(p<0.005)$.

explained in Materials and Methods, intrinsic signals make a negligible contribution to the present fluorescence measurements. Furthermore, the largest slow intrinsic signals should be observed in the directly activated cortical regions. Therefore, again, the time course relationships we observed are the opposite of those to be expected from contamination by intrinsic signals.

An alternative explanation for the optically detected spread is to assume that a significant portion of the optical signal originated from potassium accumulation and the subsequent depolarization of glial cells. Although we do not exclude some contribution from glial cell depolarization, several results suggested that a glial signal was not dominant here. First, the similarity between LFPs and optical signals (c.g., Fig. 3) indicates that signals from glial cells could have contributed only a small component of the signal. Second, the rise time and especially the fall time of the signals (e.g., Fig. 2) were faster than changes in membrane potentials recorded from glial cells in response to visual stimuli. Third, Kelly and Van Essen (1974) observed that glial depolarization exhibits selectivity for the stimulus attributes in a similar fashion to that observed for neurons in the visual cortex. If this observation is not influenced by sampling bias, then significant spread mediated by glial syncytia is not likely to occur in visual cortex. Fourth, if the detected spread 
of activity was due to electrical coupling among glial cells, the rise time of the signals detected far from their direct retinotopic activation should have been much slower. As seen in Figure $10 \mathrm{~B}$, the rise time of the response was ncarly independent of the relative cortical position. For optical signals recorded from a distant site there was usually a delay in the onset of the response.

The anatomical substrate for lateral spread. A more likely interpretation of the observed spread is that the optically detected signals indeed represent electrical activity that has spread across the cortex. Actually, this interpretation is fully consistent with the expectation of a significant spread due to the morphology of long-range intrinsic connections with their extensive clustered arbors (Fisken et al., 1975; Creutzfeldt et al., 1977; Gilbert and Wiesel, 1979, 1983, 1989; Rockland and Lund, 1982; Livingstone and Hubel, 1984; Martin and Whitteridge, 1984) as well as the extensive feedback connections from higher visual areas (e.g., Maunsell and Van Essen, 1983; Krubitzer and Kaas, 1990). Recent Biocytin injections in macaque V1 also revealed these clustered intrinsic connections of variable distance up to $2.5 \mathrm{~mm}$ (Amir et al., 1993; Malach et al., 1993). These connections and their postsynaptic targets could be the morphological substrate of the spread detected here.

Why this spread of cortical activity was missed in many of the previous investigations of macaque $V 1$ is an intriguing question. If the spread were due mostly to subthreshold postsynaptic dendritic activity or to activity in thin unmyelinated horizontal axons, then it could not be easily detected by single-unit recordings. From the basic principle underlying the detection of optical signals, discussed in Materials and Methods, and from optical imaging experiments on slices, it is clear that the optical recording technique that employs voltage-sensitive fluorescent dyes offers a very sensitive tool for detecting both synchronized activity in submicron axons (Grinvald et al., 1982a) and subthreshold dendritic activation. For example, in Figure 4 the slow component of the optical signal from a hippocampal slice, presumably reflecting EPSPs, is very large relative to the preceding fast component reflecting action potentials in CA1 neurons. In fact, in vivo optical detection of horizontal spread of activity beyond the retinotopic or somatotopic prediction has been reported in previous optical imaging experiments in the frog optic tectum (Grinvald et al., 1984) and the rat somatosensory cortex (Orbach et al., 1985).

Does the optical signal originate from presynaptic or postsynaptic cortical activity? As already explained, the optical signal emphasizes changes in membrane potential in the processes of cortical neurons rather than their cell somata. The distinction between dendritic activity and axonal activity in vivo is not straightforward, however. We believe that the contribution from dendritic activity is significantly larger than that from cortical axons. This suggestion is based on the following findings. We observed that in cat primary visual cortex optical signals recorded during ongoing activity, without a stimulus, were often as large as those obtained with an optimal visual stimulus (Grinvald et al., 1991b; Arieli, Shoham, and Grinvald, unpublished observations). This observation contradicts the expectation from single-unit recording, which exhibits the low firing rate of most cortical neurons (and their axons). Thus, action potentials probably do not make a major contribution to the optical signal. On the other hand, in those experiments the optical signals were correlated with the LFPs known to reflect postsynaptic activity (Klee et al., 1965; Elul, 1972; Creutzfeldt and Houchin, 1974).
Recently, pharmacological manipulations using cortical slices stained with $\mathrm{RH}-795$ have conclusively shown that all the long distance optical signals are postsynaptic (L. C. Katz, personal communication). Three factors may account for a larger contribution to optical signals from dendritic activity relative to that from cortical axons. First, action potentials often exhibit a large and fast positive signal followed by a much smaller but much longer negative signal (the positive and negative signals have approximately the same area). Therefore, if action potentials in axonal processes also exhibit such a time course and if the action potentials of axonal populations are not synchronized, the positive and negative signals may cancel each other. The second factor is related to the morphology of cortical cells. The extensive dendritic arbors of cortical cells are usually more compact than the intrinsic axonal arbors that spread over larger areas. Thus, optical signals originating from axons spread over larger cortical areas relative to the dendritic tree, and therefore per unit of cortical area, may be deemphasized. Third, the optical signals corresponding to action potentials from myelinated axons are not readily detectable since they originate from the nodes of Ranvier having a small membrane area relative to the myelinated regions.

Which cortical layers contributed to the dye signal? When fresh coronal slices from the in vivo stained monkey cortex (stained for 1-2 hr) were cut, we observed that the topically applied dye diffused as deep as the white matter. However, the upper layers were more fluorescent. The contribution that deep cortical layers make to the fluorescence emitted from the illuminated area depends also upon the depth to which the excitation light penetrates into these layers, and on the light scattering characteristics of the emitted fluorescence in vivo. To assess the contribution of these factors on the emitted fluorescence from different cortical layers, a glass microelectrode filled with a fluorescent dye penetrated the cortex in vivo and the emitted fluorescence was measured as a function of depth. We estimate that most $(>80 \%)$ of the fluorescence originates from the upper cortical layers (to a depth of approximately 500-700 $\mu \mathrm{m})$. Thus, the fluorescence reaching the photodetectors originates only from neuronal elements in layers I, II, and III. Although layer I is very thin, signals from layer I, whose architecture and function are not well known, might have substantially contributed to the fluorescence signal because of the large membranc arca of its finc-caliber, dense ncuronal proccss. A question still remains as to whether neuronal activity of cells in layers IV, V, and VI significantly contributes to the optical signal we detected from the upper layers. Since cell somata residing in cortical layers $\mathrm{V}$ and $\mathrm{VI}$ also possess extensive dendritic arbors in the upper layers, it is evident that the fluorescence changes emitted from the upper layers contain a contribution from cells with somata at the deepest cortical layers. Long intrinsic lateral connections have been observed in these layers (Blasdel et al., 1985; Fitzpatrick et al., 1985). Thus, in this study, the signals recorded from upper cortical layers contained some contribution also from neuronal activity of cells in the deepest cortical layers. Therefore, all of the parameters derived from the present optical measurement represent a weighted average of activity in both upper and lower layers. However, we do not know the weighting factors for each layer.

How does the spread detected here compare with that observed with other imaging techniques? In their 2-DG study, Tootell et al. (1988) observed a sharp border having a halfwidth of $140 \mu \mathrm{m}$ in the input layers. In the upper and lower 
cortical layers the spread was larger than that observed in the input layer. While quantitative data were not presented for those layers, the authors estimated that the spread of activity in layer II and layer VI was less than $1 \mathrm{~mm}$. A value of $1 \mathrm{~mm}$ is closer to the value reported here than to the value of $140 \mu \mathrm{m}$ they observed at the input layer. Furthermore, one cannot rule out that a spread much larger than $1 \mathrm{~mm}$ would have been observed in layer I and in layer II if the 2-DG images of those layers had had an improved contrast (see Fig. 18 in Tootell et al., 1988).

The anisotropy of the space constant for the activity spread. A new observation reported in this study is that the spread itself, beyond the retinotopic activation, is also anisotropic, being much larger along the axis parallel to the V1/V2 border, approximately by a factor of 2 . This axis is perpendicular to the direction of ocular dominance columns in this cortical region. Recently it has been reported that fundamental processing modules (hypercolumns) in this same cortical region are elongated, with an aspect ratio of about 2 (Bartfeld and Grinvald, 1992; see their Fig. $3 d$ showing two adjacent modules). More recent studies have shown that in macaque V1 (Yoshioka et al., 1992; Malach et al., 1993), neighboring elementary processing modules such as orientation patches may be interconnected, if they exhibit similar response properties. In addition, it has been shown that some of the intrinsic connections tend to skip one ocular dominance column, that of the contralateral eye. The cross-correlation studies by Ts'o and Gilbert (1988) also suggested such a rule for intrinsic connection between ocular dominance columns. Taken together, then, the value of the space constant for the spread along the lateral-medial axis, an axis perpendicular to the ocular dominance columns, is expected to be bigger by a factor of $\sim 2$ relative to those within the same columns. This value of 2 for the ratio is close to that found here.

Cortical surround inhibition. Our third major finding was that in the primate primary visual cortex, certain surround stimuli attenuated the response to center stimuli. We suggest but do not prove that this attenuation was caused by inhibition. With adjacent stimuli $3^{\circ}$ apart, 5-10\% attenuation was still observed. In this case the retinotopically activated cortical regions were separated by a distance of at least $6 \mathrm{~mm}$. Since surround inhibition has been found in both the retina and the LGN (McIlwain, 1966; Kruger and Fischer, 1973; Kaplan and Shapley, 1989), additional evidence was required to prove that part of the inhibition was of cortical origin. Since retinal and geniculate cells are thought to show negligible orientation selectivity (Hubel and Wiesel, 1968, 1969), the finding that the inhibition is orientation dependent suggests that at least part of the inhibition was of cortical origin.

Additional support for this idea came from an experiment in which we observed that short bars in the surround stimulus had a larger inhibitory effect than long bars on the activity evoked by a center stimulus of the same orientation. If the inhibition originated cxclusively from the retina or the LGN, one would expect exactly the opposite result; that is, long bars as a surround stimulus should cause a larger inhibitory effect. However, if a cortical origin for the inhibition is postulated, the present observation can be explained by cortical mechanisms. It is known that many cortical cells with sharp orientation tuning show endinhibition.

The four interlaced stimuli we selected for this experiment did not include a comparison between surround stimuli including either long or short bars without a center stimulus. Therefore, this set of stimuli cannot directly confirm this interpretation.
However, a recent report suggested that a major component of the end-inhibition originates from intracortical interactions (Bolz and Gilbert, 1989). Another report suggests that end-inhibition results from thalamic interactions affected by the corticogeniculate afferents (Murphy and Sillito, 1987). Both of these mechanisms depend on some cortical processing of LGN inputs.

Iso-orientation, long-range inhibition. The finding that in the primary visual cortex of primates adjacent stimuli with matched orientations produce greater suppression than stimuli with orthogonal orientations is consistent with previous reports on inhibition in cat visual cortex (Blakemore and Tobin, 1972; Maffei and Fiorentini, 1976; Hammond and MacKay, 1977; Nelson and Frost, 1978). We refer to this finding as iso-orientation inhibition or suppression. Recently, three independent studies on the primary visual cortex of primates, using single-unit recordings, 2-DG, and optical imaging, have reached similar conclusions (Grinvald et al., 1989; Van Essen et al., 1989; Born and Tootell, 1991; Knierim and Van Essen, 1992). These three studies provide a plausible link between neurophysiological data and psychophysical data obtained with human subjects. For example, Campbell and Kulikowski (1966) demonstrated that, in humans, a background grating with a certain orientation raises the detection threshold for a similarly oriented test grating. This adapting effect of the background decreased as the angle between the test and background gratings increased. Similar results have been reported by other groups (Julesz et al., 1976; Sagi and Julesz, 1985; Cannon and Fullenkamp, 1991).

In the present experiments, to avoid confusion with "endinhibition," iso-orientation inhibition was tested only when the surround stimuli were placed along an axis perpendicular to the orientation of the grating used as a center stimulus. Therefore, we do not know whether iso-orientation inhibition would be observed if the surround were to be positioned in a different location in the visual field. In related psychophysical experiments, Cannon and Fullenkamp (1991) explored this issue using vertical and horizontal bow-tic stimuli and a disk-like surround stimulus. The largest suppression was observed when the center stimulus and surround stimulus had the same orientation, and they concluded that there was a radial symmetry to the inhibitory effect. A recent single-unit study in V1 of awake monkey provided results that are fully consistent with the above psychophysical measurements (Knierim and Van Essen, 1992).

The iso-orientation suppression found in primate visual cortex in three independent studies using three different methodologies is consistent with previous interpretations of anatomical and electrophysiological results reported for cat area 18 (Matsubara et al., 1985, 1987). However, iso-orientation inhibition is not in line with the initial hypothesis that the long-range, horizontal connections between iso-orientation domains produce predominantly excitatory influences (McGuire et al., 1984; Gilbert and Wiesel, 1985, 1989, 1990; Ts'o et al., 1986; Wiesel and Gilbert, 1986). We suspect that the conflicting results and/ or interpretations reflect limitations of the methodologies used to explore the nature of long-range cortical interactions. The hypothesis that the long-range horizontal connections exert predominantly excitatory influence was based on single-unit studies, cross-correlation analysis, and electron microscopy (EM) studies, performed mostly in cat visual cortex. Since cross-correlation analysis is not a sensitive tool for detecting inhibitory interactions (Aertsen and Gerstein, 1985), the conclusion that effective inhibitory connections are not present may have to be reevaluated. Indeed, more recent EM studies of McGuire, Gil- 
bert, and Wiesel have suggested that inhibition rather than excitation may also play an important role, since it has been shown that a significant number of the excitatory synapses of the horizontal connections actually terminate on inhibitory neurons. In addition, these inhibitory neurons could have a powerful inhibitory effect on their postsynaptic targets because their axons often terminate on the neuronal somata or axonal initial segments (McGuire et al., 1991). Additional evidence for distant, inhibitory interactions was obtained using intracellular recordings from tangential cortical slices (Hirsch and Gilbert, 1991). Also relevant to this discussion are the findings of Ferster (1986, 1988), who observed directly, using in vivo intracellular recordings in cat cortex, that both inhibitory and excitatory synaptic potentials had an orientation tuning similar to the tuning of the target neuron itself. However, intracellular studies using stimuli far outside the receptive field have not yet been reported. Thus, at present we do not have direct evidence that the suppression of the center signal is caused by cortical synaptic inhibition, and optical imaging combined with in vivo injections of agonists and antagonists should be worthwhile.

Spatial resolution and clustered connections. The present experiments had excellent time resolution but a limited spatial resolution. We used a $10 \times 10$ diode array to look at a large cortical area. Each detector covered an area of 600 by $600 \mu \mathrm{m}$. At this level of spatial resolution cortical regions that exhibit sharp orientation tuning cannot be resolved by a single diode. This may account for the fact that we did not detect clustering of the horizontal connections. Thus, one should not conclude from the fuzzy nature of the single-condition optical maps (e.g., Fig. 8) that the final effect of the spread is distributed uniformly rather than in good correspondence to the patches seen in other studies (e.g., Malach et al., 1993). It is pertinent to note that since each diode monitored activity in a mixture of iso-orientation domains, the orientation-dependent suppression effects we observed were somewhat smeared. Thus, the low resolution used here may have led us to underestimate the extent of orientation-dependent suppression.

Optical measurement of cortical point-spread function. The present report demonstrates that cortical point-spread functions can be obtained using real-time optical imaging. Since cortical point-spread functions may be dynamic entities, depending on several stimulus parameters as well as the animal state, cortical point-spread functions should be explored further by mapping techniques that possess millisecond time resolution.

The slow increase of the optical signal, exhibiting a rise time of $\sim 150 \mathrm{msec}$, following the initial retinal delay, is intriguing but it was not systematically examined here. In several experiments we observed that the rise time of the signal depends on the stimulus parameters. For example, flashing stimuli elicited a faster rise time and multiple components could be detected (e.g., Fig. 2B). Such multiple components were not apparent using stationary gratings that started to drift at the onset of the "stimulus" (e.g., Figs. 7, 10). Low-contrast stimuli also produced an optical signal with multiple components (E. E. Lieke and A. Grinvald, unpublished observations). As discussed above, the optical signal does not represent spike activity in cell somata but rather depolarization of neuronal arbors. Therefore, the slow increase in the amplitude of evoked activity may reflect facilitation of the postsynaptic dendritic depolarization, and/or subthreshold depolarization resulting from reverberating activity, and/or progressively reduced inhibition, or slow depolarization by feedback connections from higher cortical areas. Our data did not permit a distinction between these alternatives, and additional exploration of this issue is required. To reconcile the time course of the increase in excitatory synaptic potentials and thc decrease often observed for PSTHs, we suggest that some of the increase in excitatory synaptic activity (as reflected by optical recording) activates inhibitory neurons. These inhibitory neurons may have a dominant effect on the firing rate of cortical neurons (as reflected by the PSTH).

Real-time optical imaging versus imaging based on slow intrinsic signals. It appears as if the two optical imaging techniques, that is, the one based on intrinsic signals and the one using voltage-sensitive dyes, may yield different results in direct imaging of the cortical point-spread function. In cortical cells the membrane area of the neuronal arborizations is estimated to be at least 1000-fold larger than the membrane area of the cell somata. Since the dye-based imaging technique emphasizes any electrical activity in the extensive neuronal arborization, real-time optical imaging should be a useful tool for studying the point-spread function of cortical input.

On the other hand, since spiking activity puts heavier metabolic demands on a cell, the imaging of the cortical output is probably more amenable to optical imaging based on intrinsic signals. Indeed, in recent optical imaging experiments based on intrinsic signals, with proper analysis, high-resolution maps that correspond nicely to cortical maps of spiking neurons were obtained (Grinvald et al., 1986, 1991 a; Frostig et al., 1990; Ts'o et al., 1990; Bonhoeffer and Grinvald, 1991, 1993). Therefore, imaging based on intrinsic signals may prove more useful for studying the cortical point-spread function of cortical outputs. It is important, however, to determine whether imaging based on intrinsic signals and imaging based on voltage-sensitive dyes indeed show a different extent of lateral spread. This is still an open issue because thus far the two techniques have not been used simultaneously to obtain retinotopic data. Furthermore, data obtained by these two techniques has been analyzed in different ways.

The use of imaging based on intrinsic signals has considerable advantages over that based on voltage-sensitive dyes. Slow measurements are technically much easier. In addition, extrinsic probes are not used and therefore photodynamic damage does not limit the duration of the imaging sessions. Thus, activity can be monitored over long periods of time and many more maps can be obtained. However, imaging based on voltagesensitive dyes is necessary if millisecond time resolution is important and if voltage changes rather than indirect metabolic signals must be monitored. Our results with the voltage-sensitive dye RH-795 are different from those in a previous report based on the voltage-sensitive dye NK-2367 (Blasdel and Salama, 1986; Blasdel, 1989, 1992). The time course for their"dyerelated signal" was very different from the time course observed in the present dye experiments, or in our previous measurements in invertebrate or mammalian preparations with NK-2367. In those earlier measurements the response time of the dye NK2367 was faster than a few milliseconds (Grinvald et al., 1977; A. Grinvald, unpublished observations in hippocampal slices). Actually, the time course reported by Blasdel and Salama for the dye NK-2367 is remarkably similar to the time course observed for intrinsic signals (compare Blasdel and Salama, 1986, Fig. 2, with Grinvald et al., 1986, Fig. $2 b$ or Frostig ct al., 1990, Fig. $3 c$ ). Therefore, one may question if the voltage-sensitive dye they used did significantly contribute to their signals. Recently, Blasdel (1992) proposed additional evidence that the 

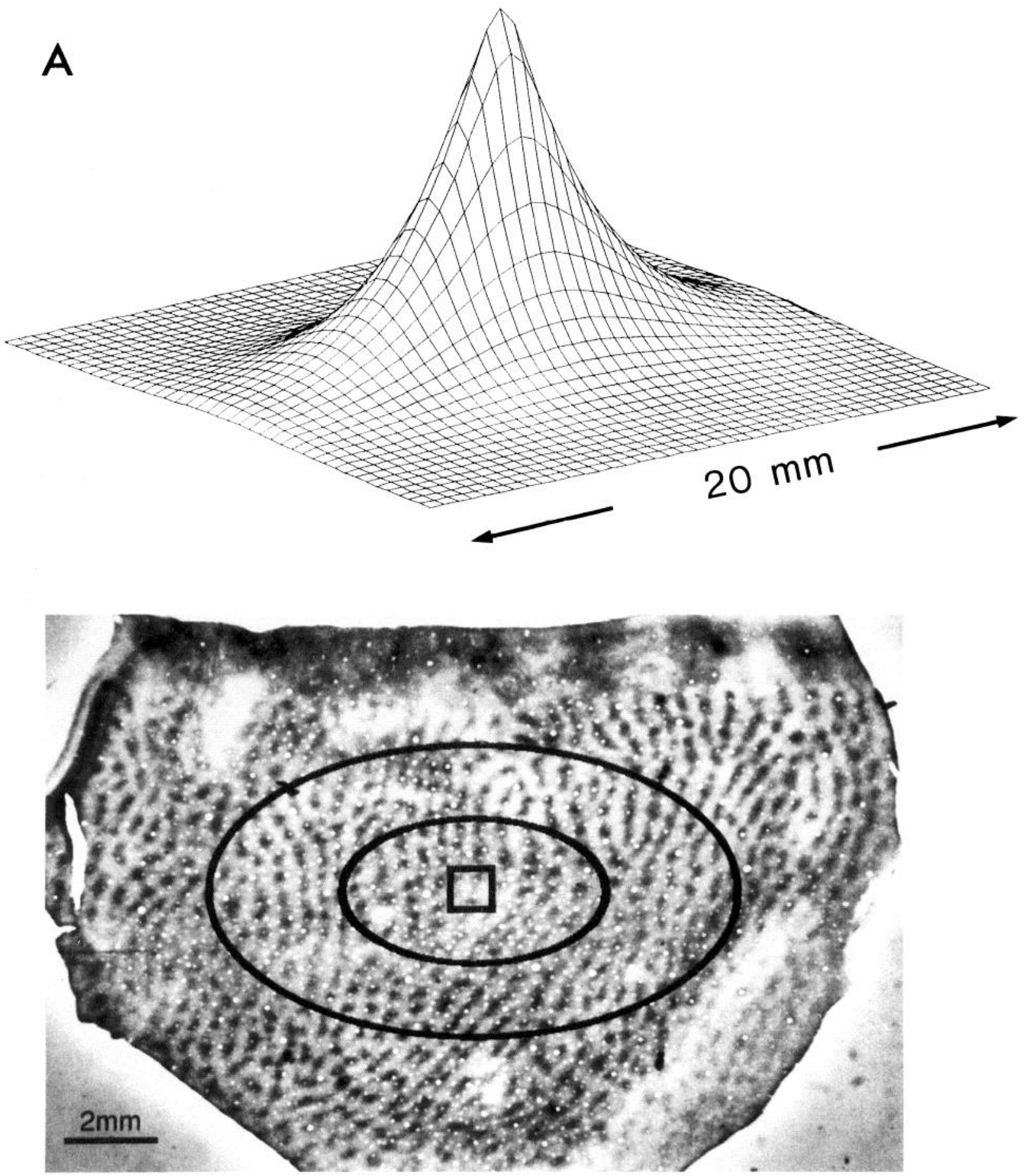

Figure 17. The number of functional domains involved in the processing of a small retinal image. $A$, Calculation of the activity spread from a small patch in layer 4 (the $1 \times 1 \mathrm{~mm}$ square) within the upper cortical layers. At an eccentricity of $\sim 6^{\circ}$, close to the V1/V2 border, such cortical activation would be produced by a retinal image of approximately $0.5^{\circ} \times 0.25^{\circ}$ presented to both eyes. The "space constants" for the exponential spread were assumed to be $1.5 \mathrm{~mm}$ and $2.9 \mathrm{~mm}$ perpendicular and parallel to the vertical meridian, respectively. To calculate the surface plot shown in $A$, these spread parameters were convolved with the $1 \times 1 \mathrm{~mm}$ square corresponding to the directly activated region. (For simplicity, exponential spread was assumed here, although the present experimental data could be fitted equally well by a Gaussian curve.) $B$, Mosaics of cytochrome oxidase blobs, close to the V1/V2 border. The thin and thick stripes of V2 are also evident in the upper part of the histological section. The center "ellipse" shows the contour where cortical activity drops to $1 / e$ (37\%) of its peak. The larger "ellipse" shows the contour where the activity drops to $1 / e^{2}(14 \%)$. More than $10,000,000$ neurons are included in the cortical area, bound by the large ellipse containing a regular mosaic of about 250 blobs. 
signal in their video imaging experiments originated from the voltage-sensitive dye they used; he found that functional images could be obtained even at the short latency of $200 \mathrm{msec}$ after the onset of the response. This argument is not compelling: it has been shown that although the intrinsic signal has a rise time of 1-2 sec, it exhibits a short onset latency of 100-150 msec after the onset of the electrical response (Grinvald et al., 1986, Fig. 2b). Thus, it is not surprising that also with high-resolution imaging based on intrinsic signals we have repeatedly observed weak but clear functional maps at latencies as short as 200-300 msec (Bonhoeffer and Grinvald, 1993; R. D. Frostig, D. Y. Ts'o, and $A$. Grinvald, unpublished observations). These apparently technical issues may prove important for the design and interpretation of optical measurements.

Activity spread and population coding in VI. The astonishing performance of the visual cortex may remain a mystery if one considers only the response properties of single cells instead of also exploring the responses of a population. To illustrate the large number of neurons and cortical modules that may process a small point of the retinal image, we calculatcd the spread of activity from a square area of 1 by $1 \mathrm{~mm}$ that was retinotopically activated in the input cortical layer. At an eccentricity of $6^{\circ} \mathrm{such}$ an area would be activated by $\sim 0.25^{\circ} \times 0.5^{\circ}$ on the retina. Figure $17 A$ shows a surface plot of the spread observed in the upper cortical layers, based on the values for the anisotropic spread observed here. To obtain this surface plot the directly activated square area was convolved with the two-dimensional exponential decay for the spread $\left(R_{x}=1.5 \mathrm{~mm}, R_{y}=3 \mathrm{~mm}\right)$. Two contours from this surface plot were then superimposed on a histological section of the cortex stained for cytochrome oxidase (Fig. 17B). This histological section serves as an example for a mosaic-like arrangement of cortical modules, blobs in this case. The central "ellipse" shows the boundaries of the area where the activity drops to $1 / e(37 \%)$ of the peak activity. The second ellipse shows the boundaries of the area where the activity drops to $1 / e^{2}(14 \%)$. More than $10,000,000$ neurons are included in this cortical area, containing regular mosaics of about 250 blobs and about 125-160 iso-orientation domains for each given orientation. These iso-orientation domains (not shown) form a patchy mosaic reminiscent of that of blobs (Blasdel and Salama, 1986; Bartfeld and Grinvald, 1992; Blasdel, 1992). This organization provides an abundant neuronal substrate for unambiguous population coding.

Conclusion. With real-time optical imaging we found that the retinotopic borders, averaged across all cortical layers, are not sharp. The space constant for the spread of cortical activity is approximately $1.5 \mathrm{~mm}$ beyond the retinotopic border at the input layer, along ocular dominance columns, but almost $3 \mathrm{~mm}$ perpendicular to the ocular dominance columns. We believe that this relationship to the functional architecture suggests that the massive spread detected here plays an important functional role. Thus, in the primary visual cortex two cortical loci that are much more than $2 \mathrm{~mm}$ from each other can "see" a portion of the activity evoked by the same single spot on the retina and may use such information for several computations. The extensive lateral spread of cortical activity that we detect raises the possibility that the degree of distributed processing (e.g., McClelland et al., 1989) in the primary visual cortex of primates is much larger than previously estimated. Optical imaging should help clarify whether a portion of the distributed processing that is assumed to take place only in higher visual areas, where the receptive fields are large, actually also plays an important role within the primary visual cortex.

\section{Appendix}

Synthesis and screening of voltage-sensitive dyes

To increase the dye diffusion in the narrow extracellular clefts of cortex and the subsequent staining of deep cortical layers, our primary effort was focused on increasing the hydrophilic properties of these dyes without losing their voltage sensitivity. The styryl dyes used here are elongated molecules measuring approximately $20-24 \AA$ in length. They are composed of two chromophores connected by a conjugated chain. An elongated substituent group is attached to each of the chromophores. One substituent is a charged hydrophilic chain. The second substituent, on the other side of the dye, is usually a hydrophobic residue, used to anchor the dyes by hydrophobic interactions with the lipid bilayer chains. It is believed that upon binding of the dye to the cell membrane, this hydrophobic chain is anchored at the lipid bilayer, together with the conjugated chromophores. The charged chain, however, is probably not embedded in the lipid bilayer but sticks out facing the extracellular space.

We synthesized more than 40 new voltage-sensitive dyes including RH-795. At the conclusion of the screening tests it was determined that the dye designated RH-795 was the optimal voltage-sensitive dye (from among the group of 40). Its chemical name is $N$-(3-dimethyl ethanol ammonium-2-hydroxy propyl)4-(4-p-diethyl aminophenyl) 1'3'-butadienyl pyridinium acetate. Thus, the most successful chemical modification of the parent compound $\mathrm{RH}-414$ seemed to be the incorporation of hydroxyl groups, on the charged chain, which further increased its hydrophilic properties. While RH-795 with two hydroxyl groups on the chain seemed to provide the best performance, two other new styryl dyes seemed to be significantly better than their parcnt dyc $\mathrm{RH}-414$. The chemical structure of these three dyes is shown in Figure $18 \mathrm{~A}$.

Figure $18 B$ illustrates the general structure of styryl dyes. To improve the dye performance we attempted additional chemical modifications. Modifications of the chemical structure can include the two chromophores A and B. Six chromophores were tested. The conjugated chain connecting the chromophores included four or six conjugated carbons. Previous experience suggested that chromophores with only two carbons absorb at shorter wavelengths and do not provide large signals in cortical tissue. The substituents on the two chromophores were also modified in a systematic fashion to optimize the dye performance. The chemical structures of the dyes synthesized and tested during this study are described in Table 1.

Synthesis of $R H-795$. The dye is synthesized by condensing an aldehyde compound and a cyclic quaternary ammonium base.

Synthesis of the quaternary ammonium base. 3-Bromo 2-propanol $N, N$-dimethyl ethanol ammonium bromide is prepared following a simplification of the method described by Gray et al. (1955). Equimolar quantities of 1,3-dibromo 2-propanol and of dimethyl ethanol amine were heated at $120^{\circ} \mathrm{C}$ for $10 \mathrm{~min}$. A heavy oil was obtained, and the unreacted materials were washed out with acetone and ether. The crude reaction gave a positive Dragendorff test (Merck reagents: dyeing reagents for thin layer and paper chromatography, reagent 130 ). 
A

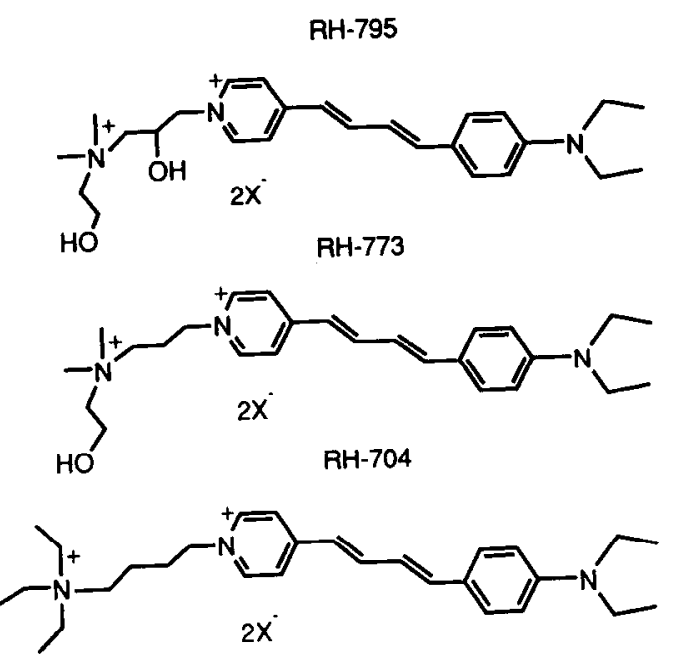

B

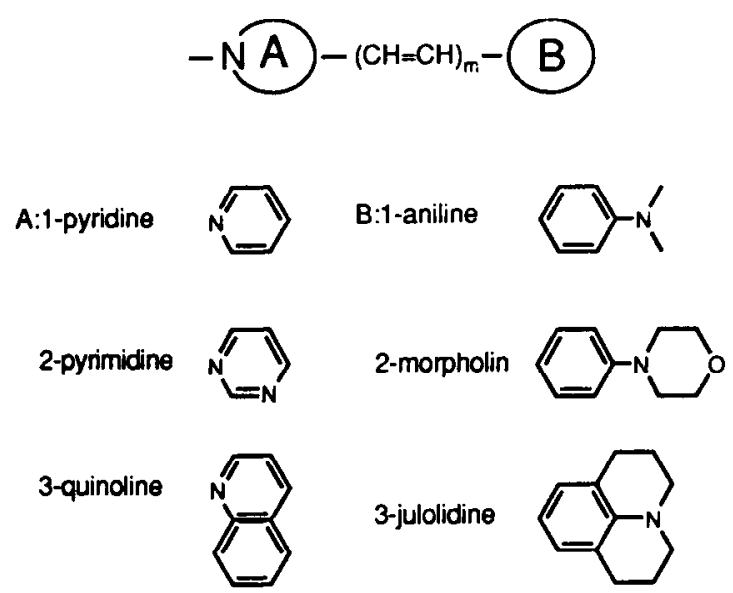

Figure 18. Chemical structures of voltage-sensitive dyes. A, Structure of the three preferred styryl dyes used in the present study. The lines show a single chemical bond between carbon, nitrogen, or oxygen atoms. Hydrogen atoms were omitted for simplicity. The length of the dyes is $20-24 \AA . B$, Chemical structures of the two chromophores of styryl dyes synthesized for this study. At the top, $A$ and $B$ show the position of the two chromophores along the conjugated carbon chain. The chemical structures of the chromophores used here are shown below. Several different substituents were attached to these chromophores, as shown in Table 1.

The resulting crude salt was found to be satisfactory for the preparation of the next product.

One equivalent each of 4-picoline and 3-bromo 2-propanol $N, N$-dimethyl ethanol ammonium bromide were heated at $120^{\circ} \mathrm{C}$ for $15 \mathrm{~min}$ and then cooled. In order to eliminate unreacted picnline, the resulting brownish gum was washed with acetone and ether. The residue was used without further purification.

Diethyl amino cinnamaldehyde was prepared as described by Jutz (1958) and Grinvald et al. (1982b). It involves the substitution of $N, N$-diethyl aniline with methyl anilino propen-1al-3, using $\mathrm{POCl}_{3}$. The desired aldehyde was purified by flash chromatography using silica gel (230-400 mesh ASTM) eluted with a mixture of hexane-ethyl acetate 9:1.

\begin{tabular}{|c|c|c|c|c|c|c|c|}
\hline & RH & $\mathrm{Y}$ & $\mathrm{n}$ & $\mathrm{m}$ & $\mathrm{L}$ & $\begin{array}{l}\text { Ab- } \\
\text { sorp- } \\
\text { tion } \\
\text { peak } \\
(\mathrm{nm})\end{array}$ & $\begin{array}{l}\text { Frac- } \\
\text { tional } \\
\text { change } \\
\left(\times 10^{-4}\right)^{g}\end{array}$ \\
\hline 1 & 808 & $\mathrm{Me}_{3} \mathrm{~N}^{+}$ & 5 & 2 & 3 & 530 & 7.2 \\
\hline 2 & 806 & $\mathrm{Me}_{3} \mathrm{~N}^{+}$ & 5 & 2 & 2 & 534 & 44 \\
\hline 3 & 829 & $\mathrm{Me}_{3} \mathrm{~N}^{+}$ & 5 & 2 & 1 & 514 & 14 \\
\hline 4 & 762 & $\mathrm{Me}_{3} \mathrm{~N}^{+}$ & $3^{\prime}$ & 2 & 3 & 534 & 4.6 \\
\hline 5 & 792 & $\mathrm{Me}_{3} \mathrm{~N}^{+}$ & $3 r$ & 2 & 2 & 532 & 36 \\
\hline 6 & 753 & $\mathrm{Me}_{3} \mathrm{~N}^{+}$ & $3^{\prime}$ & 2 & 1 & 522 & 11 \\
\hline 7 & $796^{a}$ & $\mathrm{Me}_{3} \mathrm{~N}^{+}$ & 3 & 2 & & 476 & 22 \\
\hline 8 & $837^{b}$ & $\mathrm{Me}_{3} \mathrm{~N}^{+}$ & 3 & 3 & & 576 & 14 \\
\hline 9 & $841^{\circ}$ & $\mathrm{Me}_{3} \mathrm{~N}^{+}$ & 3 & 2 & & 499 & - \\
\hline 10 & 429 & $\mathrm{Et}_{3} \mathrm{~N}^{+}$ & 4 & 2 & 4 & 540 & 12 \\
\hline 11 & 704 & $\mathrm{Et}_{3} \mathrm{~N}^{+}$ & 4 & 2 & 2 & 536 & 15 \\
\hline 12 & 414 & $\mathrm{Et}_{3} \mathrm{~N}^{+}$ & 3 & 2 & 2 & 532 & 51 \\
\hline 13 & $824^{h}$ & $\mathrm{Et}_{3} \mathrm{~N}^{+}$ & 4 & 2 & & 564 & 37 \\
\hline 14 & $830^{b, d}$ & $\mathrm{Et}_{3} \mathrm{~N}^{+}$ & 4 & 2 & & 626 & No staining \\
\hline 15 & $789^{d}$ & $\mathrm{Et}_{3} \mathrm{~N}^{+}$ & 4 & 2 & 1 & 582 & 53 \\
\hline 16 & $853^{\circ}$ & $\mathrm{F}_{1}{ }_{3} \mathrm{~N}^{+}$ & 3 & 2 & 2 & 586 & 57 \\
\hline 17 & $851^{\circ}$ & $\mathrm{Et}_{3} \mathrm{~N}^{+}$ & 3 & 2 & 1 & 572 & 38 \\
\hline 18 & 758 & $\mathrm{Et}_{3} \mathrm{~N}^{+}$ & $3^{\prime}$ & 2 & 2 & 530 & 9.3 \\
\hline 19 & $836^{h}$ & $\mathrm{SO}_{3}^{-}$ & 4 & 3 & & 566 & No optical signal \\
\hline 20 & $822^{h}$ & $\mathrm{SO}_{3}^{-}$ & 4 & 2 & & 548 & 10 \\
\hline 21 & $840^{\circ}$ & $\mathrm{SO}_{3}^{-}$ & 4 & 2 & & 484 & Not soluble \\
\hline 22 & $848^{\circ}$ & $\mathrm{SO}_{3}^{-}$ & 4 & 2 & 2 & 573 & 21 \\
\hline 23 & $845^{\circ}$ & $\mathrm{SO}_{3}^{-}$ & 4 & 2 & 1 & 560 & 50 \\
\hline 24 & 828 & $\mathrm{EtOH}_{3} \mathrm{~N}^{+}$ & 3 & 2 & 3 & 534 & - \\
\hline 25 & 800 & $\mathrm{EtOH}_{3} \mathrm{~N}^{+}$ & 3 & 2 & 2 & 532 & 42 \\
\hline 26 & 799 & $\mathrm{EtOH}_{3} \mathrm{~N}^{+}$ & 3 & 2 & 1 & 516 & 12 \\
\hline 27 & 773 & $\mathrm{Me}_{2}, \mathrm{EtOHN}^{+}$ & 3 & 2 & 2 & 536 & 24 \\
\hline 28 & 771 & $\mathrm{Me}_{2}, \mathrm{EtOHN}^{+}$ & 3 & 2 & 1 & 514 & 30 \\
\hline 29 & 795 & $\mathrm{Me}_{2}, \mathrm{EtOHN}^{+}$ & $3^{\prime}$ & 2 & 2 & 534 & 42 \\
\hline 30 & 775 & $\mathrm{Me}_{2}, \mathrm{EtOHN}^{+}$ & $3^{\prime}$ & 2 & 1 & 516 & 30 \\
\hline 31 & $823^{h}$ & $\mathrm{Me}_{2}, \mathrm{EtOHN}^{+}$ & 3 & 2 & & 564 & 28 \\
\hline 32 & 805 & $\mathrm{EtOH}_{2}, \mathrm{MeN}^{+}$ & 3 & 2 & 2 & 534 & 38 \\
\hline 33 & 804 & $\mathrm{EtOH}_{2}, \mathrm{MeN}^{+}$ & 3 & 2 & 1 & 522 & 26 \\
\hline 34 & 785 & $\mathrm{Et}_{2}, \mathrm{EtOHN}^{+}$ & 3 & 2 & 3 & 536 & 12 \\
\hline 35 & 781 & $\mathrm{Et}_{2}, \mathrm{EtOHN}^{+}$ & 3 & 2 & 2 & 534 & 54 \\
\hline 36 & 780 & $\mathrm{Et}_{2}, \mathrm{EtOHN}^{+}$ & 3 & 2 & 1 & 520 & 37 \\
\hline 37 & 811 & $\mathrm{EtOH}_{2}, \mathrm{EtN}^{+}$ & 3 & 2 & 2 & 536 & 13 \\
\hline 38 & 810 & $\mathrm{EtOH}_{2}, \mathrm{EtN}^{+}$ & 3 & 2 & 1 & 524 & 11 \\
\hline
\end{tabular}

"Di alkyl aniline was replaced by morpholino aniline.

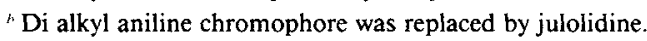

Di alkyl aniline was replaced by 2,2-dichloroethyl aniline.

"Pyridinium moiety was replaced by quinolinium.

Pyridinium was replaced by pyrimidinium.

'2-OH.

* Fractional change in fluorescence obtained in slices.

Condensation. For dye coupling, the quaternary base and the aldehyde, $1 \mathrm{~mm}$ of each, were dissolved in $5 \mathrm{ml}$ of methanol and $0.1 \mathrm{ml}$ of piperidine was added as a condensing agent. The mixture was purified by flash chromatography of silica gel. Acetonitrile and $\mathrm{H}_{2} \mathrm{O}$ were used as eluants. The magenta dye (on thin-layer chromatography) was collected. The hygroscopic dye 
was isolated as acetate salt. Its absorption maximum was 533 $\mathrm{nm}$ in absolute ethanol and $503 \mathrm{~nm}$ in $\mathrm{H}_{2} \mathrm{O}$; its fluorescence maximum was $724 \mathrm{~nm}$ in $\mathrm{H}_{2} \mathrm{O}$ (uncorrected; Shimadzu, Rf540).

\section{References}

Aertsen AMHJ, Gerstein G (1985) Evaluation of neuronal connectivity; sensitivity of cross correlation. Brain Res 340:341-354.

Albus K (1975) A quantitative study of the projection area of the central and paracentral visual field in area 17 of the cat. II. The spatial organization of the orientation domain. Exp Brain Res 24:181-202.

Allman J, Miezin F, McGuinnes E (1985) Direction and velocity specific responses from beyond the classical receptive field in the middle temporal visual field area (MT). Perception 14:105-126.

Amir Y, Harel M, Malach R (1993) Cortical hierarchy reflected in the organization of intrinsic connections in macaque monkey visual cortex. J Comp Neurol 334:19-46.

Bartfeld E, Grinvald A (1992) Relationships between orientation-preference pinwheels, cytochrome oxidase blobs, and ocular dominance columns in primate striate cortex. Proc Natl Acad Sci USA 89:1 190511909.

Blakemore CO, Tobin EA (1972) Lateral inhibition between orientation detectors in the cat's visual cortex. Brain Res 15:439-440.

Blasdel GG (1989) Visualization of neuronal activity in monkey striate cortex. Annu Rev Physiol 51:561-581.

Blasdel GG (1992) Differential imaging of ocular dominance and orientation selectivity in monkey striate cortex. J Neurosci 12:31153138.

Blasdel GG, Salama G (1986) Voltage-sensitive dyes reveal a modular organization in monkey striate cortex. Nature 321:579-585.

Blasdel GG, Lund JS, Fitzpatrick D (1985) Intrinsic connections of Macaque striate cortex: axonal projections of cells outside lamina 4C. J Neurosci 5:3350-3369.

Bolz J, Gilbert CD (1986) Generation of end-inhibition in the visual cortex via inter laminar connections. Nature 320:362-365.

Bolz J, Gilbert CD (1989) The role of horizontal connections in generating long receptive fields in the cat visual cortex. Eur J Neurosci $1: 263-268$.

Bonhoeffer T, Grinvald A (1991) Iso-orientation domains in cat visual cortex are arranged in pinwheel like patterns. Nature 353:429-431.

Bonhoeffer T, Grinvald A (1993) The layout of iso-orientation domains in area 18 of cat visual cortex: optical imaging reveals pinwheellike organization. J Neurosci 13:4157-4180.

Born RT, Tootell RBH (1991) Single-unit and 2-deoxyglucose studies of side inhibition in macaque striate cortex. Proc Natl Acad Sci USA 88:7071-7075.

Brooks B, Jung R (1973) Neuronal physiology of the visual cortex. In: Handbook of sensory physiology, Vol VII/3, Central processing of visual information (Jung R, ed), pp 325-440. New York: Springer.

Cannon MW, Fullenkamp SC (1991) Spatial interactions in apparent contrast: inhibitory effects among grating patterns of different spatial frequency, spatial positions and orientations. Vision Res 31:19851998.

Campbell FW, Kulikowski JJ (1966) Orientation selectivity of the human visual system. J Physiol (Lond) 187:437-446.

Cleland BG, Dubin MW, Levik WR (1971) Sustained and transient neurons in the cat's retina and lateral geniculate nucleus. J Physiol (Lond) 217:473-496.

Cohen LB, Lesher S (1986) Optical monitoring of membrane potential: methods of multisite optical measurement. Soc Gen Physiol Ser 40: $71-99$.

Cohen LB, Salzberg BM, Davila HV, Ross WN, Landowne D, Wagonner AS, Wang CH (1974) Changes in axon fluorescence during activity: molecular probes of membrane potential. J Membr Biol 19:1-36.

Creutzfeldt OD, Houchin J (1974) Neuronal basis of EEG waves. In: Handbook of electroencephalography and clinical neurophysiology, Vol 2C (Creutzfeldt OD, ed), pp 5-54. New York: Elsevier.

Creutzfeldt OD, Poeppel E, Singer W (1971) Quantitative Ansatz zur Analyse der funktionellen Organisation des visuellen Cortex (Untersuchungen an Primaten). In: Kybernetik Kongress, Vol 4 (Grusser OJ, ed), pp 81-96. New York: Springer.

Creutzfeldt OD, Garey LJ, Kuroda R, Wolff JR (1977) The distribution of degenerating axons after small lesions in the intact and isolated visual cortex of the cat. Exp Brain Res 27:419-440.

Daniel PM, Whitteridge D (1961) The representation of the visual field on the cerebral cortex in monkeys. J Physiol (Lond) 159:203221.

DeWeer P, Salzberg BM, eds (1986) Society of General Physiologists series, Vol 40, Optical methods in cell physiology. New York: Wiley.

Douglas RG, Martin KAC, Whitteridge D (1991) An intracellular analysis of the visual responses of neurons in cat visual cortex. $J$ Physiol (Lond) 440:659-696.

Dow BM, Snyder AA, Vautin RG, Bauer R (1981) Magnification factor and receptive field size in foveal striate cortex of the monkey. Exp Brain Res 44:213-228.

Dow BM, Vautin RG, Bauer R (1985) The mapping of visual space onto foveal striate cortex in the macaque monkey. J Neurosci 5:890 902.

Elul R (1972) The genesis of the EEG. Int Rev Neurobiol 15:228272.

Felleman DJ, Van Essen DC (1991) Distributed hierarchical processing in the primate cerebral cortex. Cereb Cortex 1:1-47.

Ferster D (1986) Orientation selectivity of synaptic potentials in neurons of cat primary visual cortex. J Neurosci 6:1284-1302.

Ferster D (1988) Spatially opponent excitation and inhibition in simple cells of the cat visual cortex. J Neurosci 8:1172-1180.

Ferster D, Koch C (1987) Neuronal connections underlying orientation selectivity in cat visual cortex. Trends Neurosci 10:487-492.

Fischer B (1973) Overlap of receptive field centers and representation of the visual field in the cat's optic tract. Vision Res 13:2113-2120.

Fisken RA, Garey CJ, Powell TPS (1975) The intrinsic, association and commissural connections of area 17 of the visual cortex. Trans R Soc London [Biol] 272:487-536.

Fitzpatrick D, Lund JS, Blasdel GG (1985) Intrinsic connections of Macaque striate cortex: afferent and efferent connections of lamina 4C. J Neurosci 5:3329-3349.

Fries W, Albus K, Creutzfeldt OD (1977) Effects of interacting visual patterns on single cell responses in cat's striate cortex. Vision Res 17: $1001-1008$

Frost JD (1968) EEG-intracellular potential relationships between EEG activity and single unit discharges in isolated cerebral cortex. Exp Neurol 24:434-443.

Frostig RD, Lieke EE, Ts'o DY, Grinvald A (1990) Cortical functional architecture and local coupling between neuronal activity and the micro circulation revealed by in vivo high-resolution optical imaging of intrinsic signals. Proc Natl Acad Sci USA 87:6082-6086.

Gilbert CD, Wiesel TN (1979) Morphology and intracortical projections of functionally identified neurons in cat visual cortex. Nature 280:120-125.

Gilbert CD, Wiesel TN (1983) Clustered intrinsic connections in cat visual cortex. J Neurosci 3:1116-1133.

Gilbert CD, Wiesel TN (1985) Intrinsic connectivity and receptive field properties in visual cortex. Vision Res 25:365-374.

Gilbert CD, Wiesel TN (1989) Columnar specificity of intrinsic horizontal and corticocortical connections in cat visual cortex. J Neurosci 9:2432-2442.

Gilbert CD, Wiesel TN (1990) The influence of contextual stimuli on the orientation selectivity of cells in primary visual cortex of the cat. Vision Res 30:1689-1701.

Gray AP, Schlieper DC, Spinner EE, Cavallito CJ (1955) The preparation of some $w$-bromoalkyl quaternary ammonium salts. J Am Chem Soc 77:3648-3649.

Grinvald A, Salzberg BM, Cohen LB (1977) Simultaneous recording from several neurones in an invertebrate central nervous system. Nature 268:140-142.

Grinvald A, Cohen LB, Lesher S, Boyle MB (1981) Simultaneous optical monitoring of activity of many neurons in invertebrate ganglia, using a 124 element 'photodiode' array. J Neurophysiol 45:829-840.

Grinvald A, Hildesheim R, Farber IC, Anglister L (1982a) Improved fluorescence probes for the measurement of rapid changes in membrane potential. Biophys J 39:301-308.

Grinvald A, Manker A, Segal M (1982b) Visualization of the spread of electrical activity in rat hippocampal slices by voltage sensitive optical probes. J Physiol (Lond) 333:269-291.

Grinvald A, Fine A, Farber IC, Hildesheim R (1983) Fluorescence monitoring of electrical responses from small neurons and their processes. Biophys J 42:195-198.

Grinvald A, Anglister L, Freeman JA, Hildesheim R, Manker A (1984) Real-time optical imaging of naturally evoked electrical activity in the intact frog brain. Nature 308:848-850. 
Grinvald A, Lieke EE, Frostig RD, Gilbert CD, Wiesel TN (1986) Functional architecture of cortex revealed by optical imaging of intrinsic signals. Nature 324:361-364.

Grinvald A, Frostig RD, Lieke EE, Hildesheim R (1988) Optical imaging of neuronal activity. Physiol Rev 68:1285-1366.

Grinvald A, Ts'o DY, Frostig RD, Lieke EE, Arieli A, Hildesheim R (1989) Optical imaging of neuronal activity in the visual cortex. In: Neural mechanisms of visual perception (Lam DMK, Gilbert CD, eds), pp 117-136. The Woodlands, TX: Portfolio.

Grinvald A, Frostig RD, Bartfeld E, Siegal RM (1991a) High resolution optical imaging of neuronal activity in awake monkey. Proc Natl Acad Sci USA 88:11559-11563.

Grinvald A, Bonhoeffer T, Malonek D, Shoham D, Bartfeld E, Arieli A, Hildesheim R, RatzlaffE (1991b) Optical imaging of architecture and function in the living brain. In: Memory, organization and locus of change (Squire L, ed), pp 49-85. New York: Oxford UP.

Hammond P, MacKay DM (1977) Differential responsiveness of simple and complex cells in cat striate cortex to visual texture. Exp Brain Res 30:275-296.

Hammond P, MacKay DM (1981) Modulatory influence of moving textured backgrounds on responsiveness of simple cells in feline striate cortex. J Physiol (Lond) 319:431-442.

Hata Y, Tsumoto T, Sato H, Hagihara K, Tamura H (1988) Inhibition contributes to orientation selectivity in visual cortex of cat. Nature 335:815-817.

Hirsch JA, Gilbert CD (1991) Synaptic physiology of horizontal connection in primary visual cortex. J Neurosci 11:1800-1809.

Hubel DH, Wiesel TN (1968) Receptive fields and functional architecture of monkey striate cortex. J Physiol (Lond) 195:215-243.

Hubel DH, Wiesel TN (1969) Anatomical demonstration of columns in the monkey striate cortex. Nature 221:747-750.

Hubcl DH, Wiesel TN (1974) Uniformity of monkey striate cortex: a parallel relationship between field size, scatter, and magnification factor. J Comp Neurol 158:295-306.

Jones BH (1970) Responses of single neurons in cat visual cortex to a simple and more complex stimulus. Am J Physiol 218:1102-1107.

Julesz B, Breitmeyer B, Kropfl W (1976) Binocular disparity-dependent upper-lower hemifield anisotropy and left-right hemifield isotropy as revealed by dynamic random dot stereograms. Perception 5:129-141.

Jutz C (1958) Darstellung ungesattigter aldehyde nach art der Vilsmeier-Reaktion. Chem Ber 91:850-861.

Kaplan E, Shaplcy RM (1989) Illumination of the receptive field surround controls the contrast gain of macaque $P$ retinal ganglion cells. Soc Neurosci Abstr 15:174.

Katz LC (1987) Local circuitry of identified neurons in cat visual cortex brain slices. J Neurosci 7:1223-1249.

Katz LC, Gilbert CD, Wiesel TN (1989) Local circuits and ocular dominance columns in monkey striate cortex. J Neurosci 9:13891399.

Kelly JP, Van Essen DC (1974) Cell structure and function in the visual cortex of the cat. J Physiol (Lond) 238:515-574.

Klee MF, Offenloch K, Tigges J (1965) Crosscorrelation analysis of electroencephalographic potentials and slow membrane transient. Science 147:519-521.

Knierim JJ, Van Essen DC (1992) Neural responses to static texture patterns in area $\mathrm{V} l$ of the alert macaque monkey. J Neurophysiol 67 : 961-980.

Konnerth A, Orkand RK (1986) Voltage sensitive dyes measure potential changes in axons and glia of frog optic nerve. Neurosci Lett 66:49-54.

Konnerth A, Obaid A, Salzberg BN (1987) Optical recording from parallel fibers and other cell types in skate cerebellar slices in vitro. J Physiol (Lond) 393:681-702.

Krubitzer LA, Kaas JH (1990) Cortical connections of MT in four species of primates: areal, modular, and retinotopic patterns. Vis Neurosci 5:165-204.

Kruger J (1977) The shift effect in the lateral geniculate body of the rhesus monkey. Exp Brain Res 29:387-392.

Kruger J, Fischer B (1973) Strong periphery effect in cat retinal ganglion cells. Excitatory responses in $\mathrm{ON}$ - and OFF-center neurons to single grid displacements. Exp Brain Res 18:316-318.

Lcv-Ram V, Grinvald A (1986) $\mathrm{Ca}^{2+}$ and $\mathrm{K}^{+}$dependent communication between central nervous system myelinated axons and oligo- dendrocytes revealed by voltage-sensitive dyes. Proc Natl Acad Sci USA 83:6651-6655.

Li Ch-L, Ortiz-Golvin A, Chou SN, Howard SY (1960) Cortical intracellular potentials in response to stimulation of lateral geniculate body. J Neurophysiol 23:592-601.

Lieke EE, Frostig RD, Ratzlaff EH, Grinvald A (1988) Center/surround inhibitory interaction in macaque $V 1$ revealed by real-time optical imaging. Soc Neurosci Abstr 14:1122.

Lieke EE, Frostig RD, Arieli A, Ts'o DY, Hildesheim R, Grinvald A (1989) Optical imaging of cortical activity: real-time imaging using extrinsic dye signals and high resolution imaging based on slow intrinsic signals. Annu Rev Physiol 51:543-559.

Livingstone MS, Hubel DH (1984) Anatomy and physiology of a color system in the primate visual cortex. J Neurosci 4:309-356.

Loew LM (1987) Optical measurement of electrical activity. Boca Raton, FL: CRC.

Loew LM, Cohen LB, Salzberg BM, Obaid AL, Bezanilla F (1985) Charge shift probes of membrane potential. Characterization of aminostyrylpyridinum dyes on the squid giant axon. Biophys J 47:7I77.

Maffei L, Fiorentini A (1976) The unresponsive regions of visual cortical receptive fields. Vision Res 16:1131-1139.

Malach R, Amir Y, Harel M, Grinvald A (1993) Relationship between intrinsic connections and functional architecture revealed by optica imaging and in-vivo targeted biocytin injections in primate striate cortex. Proc Natl Acad Sci USA 90:10469-10473.

Martin KAC, Whitteridge D (1984) Form, function and intracortical projections of spiny neurones in the striate visual cortex of the cat. J Physiol (Lond) 353:463-504.

Matsubara JA, Cynader MS, Swindale NV, Stryker MP (1985) Intrinsic projections within visual cortex: evidence for orientation-specific local connections. Proc Natl Acad Sci USA 82:935-939.

Matsubara JA, Cynader MS, Swindale NV (1987) Anatomical properties and physiological correlates of the intrinsic connections in cat area 18. J Neurosci 7:1428-1446.

Maunsell JHR, Van Essen DC (1983) The connections of the middle temporal visual area (MT) and their relationship to a cortical hierarchy in the macaque monkey. J Neurosci 3:2563-2586.

McClelland JL, Rumelhart DE, Hinton GE (1989) The appeal of parallel distributed processing. In: Parallel distributed processing $(\mathrm{Ru}-$ melhart DE, McClelland JL, eds), pp 3-44. Cambridge, M^: MIT Press.

McGuire BA, Hammond JP, Gilbert CD, Wiesel TN (1984) Patterns of synaptic input to layer 4 of striate cortex. J Neurosci 4:3021-3033.

McGuire BA, Gilbert CD, Rivlin PK, Wiesel TN (1991) Targets of horizontal connections in macaque primary visual cortex. J Comp Neurol 305:370-392.

Mcllwain JT (1966)Some evidence concerning the physiological basis of the periphery effect in the cat retina. Exp Brain Res 1:265-271.

McIlwain JT (1975) Visual receptive fields and their images in superior colliculus of the cat. J Neurophysiol 38:219-230.

Mcllwain JT (1986) Point images in the visual system: new interest in an old idea. Trends Neurosci 9:354-358.

Murphy PC, Sillito AM (1987) Corticofugal feedback influences the generation of length tuning in the visual pathway. Nature 329:727729.

Nelson JI, Frost BJ (1978) Orientation-selective inhibition from beyond the classic visual receptive field. Brain Res 139:359-365.

Nelson JI, Frost BJ (1985) Intracortical facilitation among co-oriented, co-axially aligned simple cells in cat striate cortex. Exp Brain Res 61: $54-61$

Orbach HS (1987) Monitoring electrical activity in rat cerebral cortex. In: Optical measurement of electrical activity (Loew LM, ed), pp 115135. Boca Raton: CRC.

Orbach HS, Cohen LB (1983) Simultaneous optical monitoring of activity from many areas of the salamander olfactory bulb. A new method for studying functional organization in the vertebrate CNS. J Neurosci 3:2251-2262.

Orbach HS, Van Essen DC (1993) In vivo tracing of pathways and spatio-temporal activity patterns in rat visual cortex using voltage sensitive dyes. Exp Brain Res 94:371-392.

Orbach HS, Cohen LB, Grinvald A (1985) Optical mapping of electrical activity in rat somatosensory and visual cortex. $J$ Neurosci 5:1886-1895.

Orban GA, Gulyas B, Vogels R (1987) Influence of a moving textured 
background on direction selectivity of cat striate neurons. J Neurophysiol 57:1792-1812.

Plenz D, Aertsen A (1993) Current source density profiles of optical recording maps: a new approach to the analysis of spatio-temporal neuronal activity patterns. Eur $J$ Neurosci 5:437-448.

Ratzlaff EH, Grinvald A (1991) A tandem-lens epifluorescence macroscope: hundred-fold brightness advantage for wide field imaging. J Neurosci Methods 36:127-137.

Rockland K, Lund JS (1982) Widespread periodic intrinsic connections in the tree shrew visual cortex. Science 215:1532-1534.

Ross WN, Salzberg BN, Cohen LB, Grinvald A, Davila HV, Waggoner AS, Chang CH (1977) Changes in absorption, fluorescence, dichroism and birefringence in stained axons: optical measurement of membrane potential. J Membr Biol 33:141-183.

Sagi D, Julesz B (1985) "Where" and "what" in vision. Science 228: 1217-1219.

Salzberg BM, Davila HV, Cohen LB (1973) Optical recording of impulses in individual neurones of an invertebrate central nervous system. Nature 246:508-509.

Salzberg BM, Grinvald A, Cohen LB, Davila HV, Ross WN (1977) Optical recording of neuronal activity in an invertebrate central nervous system: simultaneous monitoring of several neurons. J Neurophysiol 40:1281-1291.

Talbot SA, Marshal WH (1941) Physiological studies on neural mechanisms of visual localization and discrimination. Am J Ophthalmol $74: 1255-1264$.

Tasaki I, Watanabe A, Sandlin R, Carnay L (1968) Changes in fluorescence, turbidity and birefringence associated with nerve excitation. Proc Natl Acad Sci USA 61:883-888.

Tootell RBH, Silverman MS, De Valois RL (1982) Deoxyglucose analysis of retinotopic organization in primate striate cortex. Science 218:902-904.

Tootell RBH, Switkes E, Silverman MS, Hamilton SL (1988) Func- tional anatomy of macaque striate cortex II: retinotopic organization. J Neurosci 8:1531-1568.

Toyama K (1991) The structure-function problem in visual cortical circuitry studied by cross correlation techniques and multi channel recordings. In: Neuronal cooperativity (Kruger J, ed), pp 5-29. New York: Springer.

Ts'o DY, Gilbert CD (1988) The organization of chromatic and spatial interactions in the primate striate cortex. J Neurosci 8:1712-1727.

Ts'o DY, Gilbert CD, Wiesel TN (1986) Relationships between horizontal interactions and functional architecture in cat striate cortex as revealed by cross correlation analysis. J Neurosci 6:1 160-1170.

Ts'o DY, Frostig RD, Lieke EE, Grinvald A (1990) Functional organization of primate visual cortex revealed by high resolution optical imaging. Science 249:417-420.

Van Essen DC, Newsome WT, Maunsel JHR (1984) The visual field representation in striate cortex of the macaque monkey. Asymmetries, anisotropies and individual variability. Vision Res 24:429-448.

Van Essen DC, DeYoe EA, Olavarria JF, Knierim JJ, Fox JM, Sagi D, Julesz B (1989) Neural responses to static and moving texture patterns in visual cortex of the macaque monkey. In: Neural mechanisms of visual perception (Lam DMK, Gilbert CD, eds), pp 137-156. The Woodlands, TX: Portfolio.

Waggoner AS, Grinvald A (1977) Mechanisms of rapid optical changes of potential sensitive dyes. Ann NY Acad Sci 303:217-242.

Wiesel TN, Gilbert CD (1986) Visual cortex. Trends Neurosci 9:509_ 512.

Yoshioka T, Blasdel GG, Levitt IB, Lund IS (1992) Patterns of lateral connections in Macaque visual area V1 revealed by biocytin histochemistry and functional imaging. Soc Neurosci Abstr 18:299.

Zeki S (1983) Color coding in the cerebral cortex: the response of wavelength selective and color coded cells in monkey visual cortex to changes in wavelength composition. Neuroscience 9:767-781. 